\title{
On Noncooperative Games, Minimax Theorems and Equilibrium Problems
}

\author{
J.B.G.Frenk and G.Kassay \\ 1 Econometric Institute, Erasmus University, Rotterdam, Netherlands \\ frenk@few. eur.nl \\ 2 Faculty of Mathematics and Computer Science, Babes-Bolyai University, Cluj, \\ Romania kassay@math.ubbcluj.ro
}

Econometric Institute Report E.I. 2006-21

\section{Introduction to noncooperative game theory}

To introduce a static two-player zero-sum (noncooperative) game (for more details and examples see [1], [2], [3], [4] or [5]) and its relation to a minimax theorem we consider two players called 1 and 2 and assume that the set of pure strategies (also called actions) of player 1 is given by some nonempty set $A$, while the set of pure strategies of player 2 is given by a nonempty set $B$. Without loss of generality we may assume that the sets $A$ and $B$ are topological spaces with Borel $\sigma$-algebras $\mathcal{B}(A)$, respectively $\mathcal{B}(B)$. By definition a Borel $\sigma$-algebra is the smallest $\sigma$-algebra generated by the open sets $([6])$. If player 1 chooses the pure strategy $a \in A$ and player 2 chooses the pure strategy $b \in B$, then player 2 has to pay player 1 an amount $f(a, b)$ with $f: A \times B \rightarrow R$ a given function. This function is called the payoff function of player 1 . Since the gain of player 1 is the loss of player 2 (this is a so called zero-sum game) the payoff function of player 2 is $-f$. Clearly player 1 likes to gain as much profit as possible. However, at the moment he does not know how to achieve this and so he first decides to compute a lower bound on his profit. To compute this lower bound player 1 argues as follows: if he decides to choose action $a \in A$, then it follows that his profit is at least $\inf _{b \in B} f(a, b)$, irrespective of the action of player 2. Therefore a lower bound on the profit for player 1 is given by

$$
r_{*}:=\sup _{a \in A} \inf _{b \in B} f(a, b) .
$$

Similarly player 2 likes to minimize his losses but since he does not know how to achieve this he also decides to compute first an upper bound on his losses. To do so, player 2 argues as follows. If he decides to choose action $b \in B$, 
it follows that he loses at most $\sup _{a \in A} f(a, b)$ and this is independent of the action of player 1 . Therefore an upper bound on his losses is given by

$$
r^{*}:=\inf _{b \in B} \sup _{a \in A} f(a, b) .
$$

Since the profit of player 1 is at least $r_{*}$ and the losses of player 2 is at most $r^{*}$ and the losses of player 2 are the profits of player 1, it follows directly that $r_{*} \leq r^{*}$. In general $r_{*}<r^{*}$, but under some properties on the pure strategy sets and payoff function one can show that $r_{*}=r^{*}$. If this equality holds and in relations (1) and (2) the suprema and infima are attained, an optimal strategy for both players is obvious. By the interpretation of $r_{*}$ for player 1 and the interpretation of $r^{*}$ for player 2 and $r^{*}=r_{*}:=v$ both players will choose an action which achieves the value $v$ and so player 1 will choose that action $a_{0} \in A$ satisfying

$$
\inf _{b \in B} f\left(a_{0}, b\right)=\max _{a \in A} \inf _{b \in B} f(a, b) .
$$

Moreover, player 2 will choose that strategy $b_{0} \in B$ satisfying

$$
\sup _{a \in A} f\left(a, b_{0}\right)=\min _{b \in B} \sup _{a \in A} f(a, b) .
$$

In case only $r_{*}=r^{*}$ or equivalently

$$
\inf _{b \in B} \sup _{a \in A} f(a, b)=\sup _{a \in A} \inf _{b \in B} f(a, b)
$$

both players can approximate their optimal pure strategies by so-called $\epsilon$ optimal pure strategies. A pure strategy $a_{0} \in A$ for player 1 is called an $\epsilon$-optimal pure strategy if

$$
\inf _{b \in B} f\left(a_{0}, b\right) \geq v-\epsilon
$$

A similar definition applies to an $\epsilon$-optimal pure strategy for player 2. By these observations it is now important to know for which payoff functions and pure strategy sets the so-called minimax result $r_{*}=r^{*}$ holds and under which conditions the supremum in relation (1) and the infimum in relation (2) are attained. Before discussing this, we give an example for which the equality $r^{*}=r_{*}$ does not hold.

Example 1. Consider the continuous payoff function $f:[0,1] \times[0,1] \rightarrow[0, \infty)$ given by

$$
f(a, b)=(a-b)^{2} .
$$

For this function it holds for every $0 \leq a \leq 1$ that $\inf _{b \in[0,1]}(a-b)^{2}=0$ and so

$$
r_{*}:=\sup _{0 \leq a \leq 1} \inf _{0 \leq b \leq 1}(a-b)^{2}=0 .
$$

Moreover, it follows that

$$
\sup _{0 \leq a \leq 1}(a-b)^{2}=(1-b)^{2}
$$


for every $0 \leq b<\frac{1}{2}$ and

$$
\sup _{0 \leq a \leq 1}(a-b)^{2}=b^{2}
$$

for every $\frac{1}{2} \leq b \leq 1$. This shows

$$
r^{*}:=\inf _{0 \leq b \leq 1} \sup _{0 \leq a \leq 1}(a-b)^{2}=4^{-1}
$$

and so $r_{*}$ does not equal $r^{*}$.

The above example shows a particular case for which it is not clear how the players should select their strategies. A possible solution to this problem is to extend the set of pure strategies to the larger set of so-called mixed strategies. Recall in the next definition that a Borel finite measure on a topological space $D$ is a finite measure defined on the Borel $\sigma$-algebra $\mathcal{B}(D)$ of $D$ (for more details on Borel measures see [7], [8], [9]). Moreover, we also need in this definition the unit simplex $\Delta_{k} \subseteq R^{k}$ given by

$$
\Delta_{k}:=\left\{\alpha^{\top}=\left(\alpha_{1}, \ldots, \alpha_{k}\right) \in R^{k}: \sum_{i=1}^{k} \alpha_{i}=1, \alpha_{i} \geq 0,1 \leq i \leq k\right\} .
$$

Definition 1. Let $D$ be a nonempty topological space and $\mathcal{B}(D)$ its Borel $\sigma$ algebra. A Borel finite measure $\epsilon_{d}: \mathcal{B}(D) \rightarrow[0, \infty)$ is called a one-point Borel probability measure concentrated on the set $\{d\}$ if $\epsilon_{d}\left(D_{0}\right)=1$ for $D_{0} \in \mathcal{B}(D)$ containing $d$ and $\epsilon_{d}\left(D_{0}\right)=0$ otherwise. A Borel finite measure $\nu: \mathcal{B}(D) \rightarrow$ $[0,1]$ is called a Borel probability measure with finite support if there exists some finite set $\left\{d_{1}, \ldots, d_{k}\right) \subseteq D$ and some vector $s(\nu)^{\top} \in \Delta_{k}$ with $s_{i}(\nu)>$ $0,1 \leq i \leq k$ such that

$$
\nu=\sum_{i=1}^{k} s_{i}(\nu) \epsilon_{d_{i}} .
$$

If we denote by $\mathcal{P}_{F}(D)$ the set of all Borel probability measures on $D$ with a finite support, then within game theory any element $\nu$ belonging to $\mathcal{P}_{F}(D)$ is called a mixed strategy and it has the following interpretation. If a player with pure strategy set $D$ selects the mixed strategy

$$
\nu=\sum_{i=1}^{k} s_{i}(\nu) \epsilon_{d_{i}},
$$

then with probability $s_{i}(\nu), 1 \leq i \leq k$ this player will use the pure strategy $d_{i} \in D$. By this interpretation it is clear that the set $D$ of pure strategies can be identified within the set of mixed strategies by the one-point Borel probability measures $\left\{\epsilon_{d}: d \in D\right\}$. We now assume that player 1, respectively player 2 are using their sets of mixed strategies. This means that the payoff function $f$ should be extended to a function $f_{e}: \mathcal{P}_{F}(A) \times \mathcal{P}_{F}(B) \rightarrow R$. This extension is defined by

$$
f_{e}(\lambda, \mu):=\sum_{i=1}^{k} \sum_{j=1}^{m} s_{i}(\lambda) s_{j}(\mu) f\left(a_{i}, b_{j}\right)
$$


with $\lambda=\sum_{i=1}^{k} s_{i}(\lambda) \epsilon_{a_{i}} \in \mathcal{P}_{F}(A)$ and $\mu=\sum_{j=1}^{m} s_{j}(\mu) \epsilon_{b_{j}} \in \mathcal{P}_{F}(B)$ and it represents the expected profit for player 1 or expected loss of player 2 if player 1 selects the mixed strategy $\lambda \in \mathcal{P}_{F}(A)$ and player 2 selects the mixed strategy $\mu \in \mathcal{P}_{F}(B)$. Under some topological/algebraic conditions on the function $f$ and the sets $A$ and $B$ of pure strategies it can be shown that the game represented by $f_{e}$ and the mixed strategy sets $\mathcal{P}_{F}(A)$ and $\mathcal{P}_{F}(B)$ has a solution. This means that we need to investigate under which necessary and sufficient conditions the following minimax result holds

$$
\inf _{\mu \in \mathcal{P}_{F}(B)} \sup _{\lambda \in \mathcal{P}_{F}(A)} f_{e}(\lambda, \mu)=\sup _{\lambda \in \mathcal{P}_{F}(A)} \inf _{\mu \in \mathcal{P}_{F}(B)} f_{e}(\lambda, \mu) .
$$

In case player 2 is only allowed to use his pure strategy set $B$, we will also investigate under which necessary and sufficient conditions the game represented by $f_{e}$ and the sets $B$ and $\mathcal{P}_{F}(A)$ has a solution. Hence for this case we need to check under which conditions the minimax result

$$
\inf _{b \in B} \sup _{\lambda \in \mathcal{P}_{F}(A)} f_{e}\left(\lambda, \epsilon_{b}\right)=\sup _{\lambda \in \mathcal{P}_{F}(A)} \inf _{b \in B} f_{e}\left(\lambda, \epsilon_{b}\right)
$$

holds. Finally, if player 1 and player 2 are only allowed to use their pure strategy sets we again pose the same question and investigate under which necessary and sufficient conditions the game represented by $f$ and the sets $A$ and $B$ has a solution or equivalently under which condition the classical minimax result

$$
\inf _{b \in B} \sup _{a \in A} f(a, b)=\sup _{a \in A} \inf _{b \in B} f(a, b) .
$$

holds. A slight extension of a two player zero-sum game is given by a so-called two player constant-sum game. In this case each player has a payoff function $f_{i}, i=1,2$ and for these payoff functions there exists some $c \in R$ such that

$$
f_{1}(a, b)+f_{2}(a, b)=c
$$

for every $a \in A$ and $b \in B$. As in a zero-sum game the gain for player 1 , respectively player 2 is given by $f_{1}(a, b)$, respectively $f_{2}(a, b)$ when both players select independently the strategies $a$, respectively $b$. Introducing for this game the payoff functions $\widetilde{f}_{i}, i=1,2$ given by

$$
\tilde{f}_{i}=f_{i}-c_{i}
$$

with $c_{1}+c_{2}=c$ it is easy to see that the analysis of the original constant sum game reduces to the analysis of a zero-sum game with payoff function $\widetilde{f}_{1}$ for player 1 . The above two player zero-sum (constant-sum) noncooperative game can also be extended to a nonconstant-sum noncooperative game involving $n \geq 2$ players. In this model we have $n$ players, $n \geq 2$ and player $i$, $1 \leq i \leq n$ has a pure strategy set $X_{i}$ and a payoff function $f_{i}: X \rightarrow R$ with $X=\Pi_{i=1}^{n} X_{i}$ (for a detailed definition of these games the reader is referred to [1], [3] or [10]) Embedding the two player zero-sum game into this more 
general framework we observe that in this case player 1 has payoff function $f_{1}=f$, while player 2 has payoff function $f_{2}=-f$. For the nonconstant-sum case and $n \geq 2$ we use the notation $X_{i}$ to distinguish between the two different models and as before the pure strategy sets $X_{i}, i=1, \ldots, n$ are topological spaces. For the more general $n$-player nonzero-sum noncooperative games the concept of a minimax or saddle point approach used within a two player zerosum game is generalized and replaced by a so-called Nash equilibrium point ([12], [13]). In Section 6 these more general games will be explained in detail. To analyse the minimax relations given in (6) up to (8) for a two player zero-sum (noncooperative) game we start in Section 2 with a discussion of Wald's minimax theorem. This theorem plays a key role in deriving necessary and sufficient conditions and will be proved using two different methods. The first proof uses the separation result of disjoint convex sets in convex analysis, while the second one uses strong linear programming duality and some elementary properties of compact sets. In Section 3 these conditions together with an overview of important sufficient conditions which have appeared in the literature are discussed. Also we show that the sufficient conditions discussed in the literature can be easily verified using our necessary and sufficient conditions. In Section 4 we then give the relations between the different minimax theorems, while in Section 5 we consider the famous minimax result of the form (8) derived by Sion ([14]). Unfortunately, it remains an open question whether this minimax result can be derived directly from our necessary and sufficient conditions discussed in Section 3. Although it is not well known, a primitive version of Sion's minimax theorem already appeared in the classical paper by von Neumann ([15],[16]). The proof of Sion's theorem given here is completely elementary and uses a proof technique originated by Joó ([17], [18]) which differs from the original proof using the so-called KKM (KnasterKuratowski-Mazurkiewicz) lemma. Observe the KKM lemma is equivalent to the Brouwer fixed point theorem ([19]) and is discussed in Section 6. Also in Section 6 we introduce the extension of a two player zero-sum game to a $n$-player nonzero-sum (noncooperative) game and introduce the concept of a Nash equilibrium point. Moreover, we prove that under certain conditions a $n$-player nonzero-sum (noncooperative) game indeed has a Nash equilibrium point using a simple proof which applies the aforementioned KKM lemma. Unfortunately it remains an open question whether it is possible to prove the existence of a Nash equilibrium point by the elementary techniques used for the two player zero-sum model.

\section{On Wald's minimax theorem}

We assume in this section that the reader is familiar with the basic notions in set theory, analysis and some elementary function theory (for more details see [20]). Besides this basic knowledge this section will be self contained. To show for the different minimax results listed in relation (6) up to (8) 
necessary and sufficient conditions on the payoff function $f$ and the sets $A$ and $B$, we first need to discuss in detail Wald's minimax theorem and this will be the topic of this section. The derivation of the necessary and sufficient conditions will be postponed until Section 3. For readers familiar with convex analysis a proof of Wald's minimax theorem will be given using the (finite dimensional) separating hyperplane result, while for readers more familiar with linear programming we will show Wald's minimax result using the strong duality theorem of linear programming and some elementary properties of compact sets. We first start with a proof using tools from convex analysis. To do so, we first need to recall some well known definitions and introduce the proper notation.

Definition 2. $A$ subset $C$ of a linear space is called convex if for every $0<$ $\beta<1$ and $x, y \in C$ it follows that $\beta x+(1-\beta) y$ belongs to $C$.

In set notation this means that $\beta C+(1-\beta) C \subseteq C$ for every $0<\beta<1$.

Definition 3. A real-valued function $k: C \rightarrow R$ is called convex on the (convex) subset $C$ if

$$
k(\beta x+(1-\beta) y) \leq \beta k(x)+(1-\beta) k(y)
$$

for every $0<\beta<1$ and $x, y \in C$ and it is called concave on $C$ if $-k$ is convex. The function $k: C \rightarrow R$ is called affine on $C$ if it is both convex and concave on $C$.

Introducing the set

$$
R_{-}^{n}:=\left\{x=\left(x_{1}, \ldots, x_{n}\right) \in R^{n}: x_{i} \leq 0,1 \leq i \leq n\right\}
$$

and

$$
x^{\top} y=\sum_{i=1}^{n} x_{i} y_{i}
$$

the inner product of the vectors $x^{\top}=\left(x_{1}, \ldots, x_{n}\right) \in R^{n}$ and $y^{\top}=\left(y_{1}, \ldots, y_{n}\right) \in$ $R^{n}$ (by $x^{\top}$ we denote the transpose of the column vector $x$ ) the most elementary minimax result is given by the following.

Theorem 1. If $C \subseteq R^{n}$ is a convex set, then it follows that

$$
\inf _{x \in C} \max _{\alpha \in \Delta_{n}} \alpha^{\top} x=\max _{\alpha \in \Delta_{n}} \inf _{x \in C} \alpha^{\top} x .
$$

Proof. It is obvious that

$$
\inf _{x \in C} \max _{\alpha \in \Delta_{n}} \alpha^{\top} x \geq \max _{\alpha \in \Delta_{n}} \inf _{x \in C} \alpha^{\top} x .
$$

To show that we actually have an equality in relation (9) we assume by contradiction that 
On Noncooperative Games, Minimax Theorems and Equilibrium Problems

$$
\inf _{x \in C} \max _{\alpha \in \Delta_{n}} \alpha^{\top} x>\max _{\alpha \in \Delta_{n}} \inf _{x \in C} \alpha^{\top} x:=\gamma
$$

By relation (10) there exists some $\beta$ satisfying

$$
\inf _{x \in C} \max _{\alpha \in \Delta_{n}} \alpha^{\top} x>\beta>\gamma
$$

Introduce now the mapping $H: C \rightarrow R^{n}$ given by

$$
H(x):=x-\beta e
$$

with $e^{\top}=(1,1, \ldots, 1) \in R^{n}$. If the set $H(C) \cap R_{-}^{n}$ is nonempty, there exists some $x_{0} \in C$ satisfying $x_{0}-\beta e \leq 0$. This implies $\max _{\alpha \in \Delta_{n}} \alpha^{\top} x_{0} \leq \beta$ and we obtain a contradiction with relation (11). Hence the set $H(C) \cap R_{-}^{n}$ is empty and by the separation result for disjoint convex sets ([11]) one can find some $\alpha_{0} \in \Delta_{n}$ satisfying $\inf _{x \in C} \alpha_{0}^{\top} x \geq \beta$. This implies by the definition of $\gamma$ that $\gamma \geq \inf _{x \in C} \alpha_{0}^{\top} x \geq \beta$ contradicting relation (11) and the desired result is proved.

Let us introduce the following notation. The set $\mathcal{F}\left(A_{0}\right)$ represents the set of all finite subsets of the set $A_{0} \subseteq A$ and for every $I \in \mathcal{F}\left(A_{0}\right)$ the set $\mathcal{P}(I)$ denotes the set of all Borel probability measures concentrated on $I$. This means for $I=\left\{a_{1}, \ldots, a_{|I|}\right\} \subseteq A$ and $|I|<\infty$ denoting the cardinality of the set $I$ that $\lambda$ belongs to $\mathcal{P}(I)$ if and only if

$$
\lambda=\sum_{i=1}^{|I|} s_{i}(\lambda) \epsilon_{a_{i}}
$$

for some $s(\lambda)^{\top} \in \Delta_{|I|}$. By relation (12) it is clear that the set $\mathcal{P}(I)$ is convex and in particular

$$
\mathcal{P}(I)=\operatorname{co}\left(\left\{\epsilon_{a}\right\}_{a \in I}\right)
$$

with $\operatorname{co}(C)$ denoting the convex hull of a set $C$. Remember $\operatorname{co}(C)$ represents the set of all finite convex combinations of elements of the set $C([11])$. By the definition of $\mathcal{P}_{F}\left(A_{0}\right)$ with $A_{0} \subseteq A$ we also obtain that

$$
\mathcal{P}_{F}\left(A_{0}\right)=\operatorname{co}\left(\left\{\epsilon_{a}\right\}_{a \in A_{0}}\right)=\cup_{I \in \mathcal{F}\left(A_{0}\right)} \mathcal{P}(I)
$$

and this set is also convex. In the next theorem we will prove Wald's minimax result. This result was proved in 1945 ([21]) using a more complicated approach.

Theorem 2. For any payoff function $f: A \times B \rightarrow R$ and every set $I$ belonging to $\mathcal{F}(A)$

$$
\inf _{\mu \in \mathcal{P}_{F}(B)} \max _{\lambda \in \mathcal{P}(I)} f_{e}(\lambda, \mu)=\max _{\lambda \in \mathcal{P}(I)} \inf _{\mu \in \mathcal{P}_{F}(B)} f_{e}(\lambda, \mu) .
$$

Proof. Let $I$ belong to $\mathcal{F}(A)$ and introduce the mapping $L: \mathcal{P}_{F}(B) \rightarrow R^{|I|}$ given by

$$
L(\mu):=\left(f_{e}\left(\epsilon_{a}, \mu\right)\right)_{a \in I}
$$


By the definition of the mapping $L$ and the function $f_{e}$ we obtain

$$
\inf _{\mu \in \mathcal{P}_{F}(B)} \max _{\lambda \in \mathcal{P}(I)} f_{e}(\lambda, \mu)=\inf _{x \in L\left(\mathcal{P}_{F}(B)\right)} \max _{s(\lambda) \in \Delta_{|I|}} s(\lambda)^{\top} x
$$

and

$$
\max _{\lambda \in \mathcal{P}(I)} \inf _{\mu \in \mathcal{P}_{F}(B)} f_{e}(\lambda, \mu)=\max _{s(\lambda) \in \Delta_{|I|}} \inf _{x \in L\left(\mathcal{P}_{F}(B)\right)} s(\lambda)^{\top} x .
$$

Also by relation (5) it follows for every $a \in I$ that the function

$$
\mu \longmapsto f_{e}\left(\epsilon_{a}, \mu\right)
$$

is affine on $\mathcal{P}_{F}(B)$. This shows by the convexity of the set $\mathcal{P}_{F}(B)$ that the range $L\left(\mathcal{P}_{F}(B)\right) \subseteq R^{|I|}$ is a convex set. Applying now Theorem 1 we obtain

$$
\inf _{x \in L\left(\mathcal{P}_{F}(B)\right)} \max _{s(\lambda) \in \Delta_{|I|}} s(\lambda)^{\top} x=\max _{s(\lambda) \in \Delta_{|I|}} \inf _{x \in L\left(\mathcal{P}_{F}(B)\right)} s(\lambda)^{\top} x,
$$

and by relations (15) and (16) the desired result follows.

A symmetrical version of Wald's minimax theorem needed in the proof of Lemma 4 is given by

$$
\sup _{\lambda \in \mathcal{P}_{F}(A)} \min _{\mu \in \mathcal{P}(J)} f_{e}(\lambda, \mu)=\min _{\mu \in \mathcal{P}(J)} \sup _{\lambda \in \mathcal{P}_{F}(A)} f_{e}(\lambda, \mu)
$$

for any $J$ belonging to $\mathcal{F}(B)$. This can be easily derived from Theorem 2 (replace $f_{e}(\lambda, \mu)$ by $-f_{e}(\lambda, \mu)$ and reverse the sets $A$ and $B$ !). Using the next lemma it is also possible to give different equivalent representations of Wald's minimax theorem.

Lemma 1. Let $f: A \times B \rightarrow R$ be a given payoff function. For any $\mu \in \mathcal{P}_{F}(B)$ and $A_{0} \subseteq A$

$$
\sup _{\lambda \in \mathcal{P}_{F}\left(A_{0}\right)} f_{e}(\lambda, \mu)=\sup _{a \in A_{0}} f_{e}\left(\epsilon_{a}, \mu\right)
$$

while for any $B_{0} \subseteq B$ and $\lambda \in \mathcal{P}_{F}(A)$

$$
\inf _{\mu \in \mathcal{P}_{F}\left(B_{0}\right)} f_{e}(\lambda, \mu)=\inf _{b \in B_{0}} f_{e}\left(\lambda, \epsilon_{b}\right) .
$$

Proof. We only give a proof of the first equality since the second one can be verified in a similar way. Since the set $A_{0} \subseteq A$ can be identified with the set of one point Borel probability measures $\epsilon_{a}, a \in A_{0}$, it is obvious for every $\mu$ belonging to $\mathcal{P}_{F}(B)$ that

$$
\sup _{\lambda \in \mathcal{P}_{F}\left(A_{0}\right)} f_{e}(\lambda, \mu) \geq \sup _{a \in A_{0}} f_{e}\left(\epsilon_{a}, \mu\right) .
$$

Consider now an arbitrary $\lambda$ belonging to $\mathcal{P}_{F}\left(A_{0}\right)$. By definition there exists a finite set $\left\{a_{1}, \ldots, a_{k}\right\} \subseteq A_{0}$ and $s(\lambda)^{\top} \in \Delta_{k}$ such that $\lambda=\sum_{i=1}^{k} s_{i}(\lambda) \epsilon_{a_{i}}$, and hence we obtain

$$
f_{e}(\lambda, \mu)=\sum_{i=1}^{k} s_{i}(\lambda) f_{e}\left(\epsilon_{a_{i}}, \mu\right) \leq \sup _{a \in A_{0}} f_{e}\left(\epsilon_{a}, \mu\right) .
$$

Since $\lambda$ belonging to $\mathcal{P}_{F}\left(A_{0}\right)$ is arbitrary this implies

$$
\sup _{\lambda \in \mathcal{P}_{F}\left(A_{0}\right)} f_{e}(\lambda, \mu) \leq \sup _{a \in A_{0}} f_{e}\left(\epsilon_{a}, \mu\right)
$$

and the desired result is verified. 
By Lemma 1 it follows with $A_{0}$ replaced by $I \in \mathcal{F}(A)$ and $\mathcal{P}_{F}\left(A_{0}\right)$ by $\mathcal{P}(I)$ that

$$
\inf _{\mu \in \mathcal{P}_{F}(B)} \max _{\lambda \in \mathcal{P}(I)} f_{e}(\lambda, \mu)=\inf _{\mu \in \mathcal{P}_{F}(B)} \max _{a \in I} f_{e}\left(\epsilon_{a}, \mu\right)
$$

By a similar argument we obtain

$$
\max _{\lambda \in \mathcal{P}(I)} \inf _{\mu \in \mathcal{P}_{F}(B)} f_{e}(\lambda, \mu)=\max _{\lambda \in \mathcal{P}(I)} \inf _{b \in B} f_{e}\left(\lambda, \epsilon_{b}\right),
$$

and combining relations (18), (19) and Theorem 2 one can give different equivalent representations of Wald's minimax theorem. For its proof using the strong duality theorem of linear programming we need some elementary properties of closed and compact sets.

Definition 4. A topological space $X$ is called compact if every collection of open subsets of $X$ which covers $X$ contains a finite subcollection covering $X$.

It is well known that $X \subseteq R^{n}$ is compact if and only if it is bounded and closed. ([20]). Moreover, an easy consequence of the above definition is the socalled finite intersection property of compact sets given by the following ([6]): any collection of closed subsets of a compact topological space $X$, for which any finite subcollection has a nonempty intersection, must have a nonempty intersection.

Definition 5. A function $k: X \rightarrow R$ with $X$ a topological space is called lower semicontinuous if all its lower level sets $\{x \in X: k(x) \leq r\}, r \in R$ are closed subsets of $X$. It is called upper semicontinuous if all its upper level sets $\{x \in X: k(x) \geq r\}, r \in R$ are closed subsets of $X$ and it is called continuous if it is both upper and lower semicontinuous.

One can now show the following so-called Weierstrass-Lebesgue lemma $([6])$. For completeness a proof is listed.

Lemma 2. If the function $k: X \rightarrow(-\infty, \infty]$ is lower semicontinuous and $X$ is a compact topological space, then the function $k$ is bounded from below and attains its minimum on $X$.

Proof. Since $k$ is a lower semicontinuous function with values $>-\infty$ it follows that the decreasing sequence $O_{n}:=\{x \in X: k(x)>n\}, n \in Z$ of open sets covers $X$. This implies by the compactness of $X$ that there exist a finite subcover and since $O_{n+1} \subseteq O_{n}$ one can find some $m \in Z$ satisfying $X \subseteq O_{m}$ and so the function $k$ is bounded from below. To show that the function $k$ attains its minimum introduce $\beta:=\inf _{x \in X} k(x)$. If $\beta=\infty$ we are done. Hence we assume that $\beta<\infty$ and by the first part $\beta$ is finite. Consider now the collection of nonempty closed sets $F_{n}=\left\{x \in X: k(x) \leq \beta+n^{-1}\right\}, n \in N$. Since $F_{n+1} \subseteq F_{n}$ it follows that by the definition of $\beta$ that $\cap_{n \in I} F_{n}$ is nonempty for every finite subset $I$ of $N$. Hence by the finite intersection property of compact sets we obtain that the intersection $\cap_{n \in N} F_{n}$ is nonempty and this shows that $k$ attains its minimum on $X$. 
A symmetrical version of the above result is given by the following. If the function $k: X \rightarrow[-\infty, \infty)$ is upper semicontinuous and $X$ is a compact topological space, then the function $k$ is bounded from above and attains its maximum on $X$. As shown by the next observation the above result is useful in determining whether an optimal pure strategy for player 2 exists if the minimax relations (7) or (8) hold. Since for any payoff function $f: A \times B \rightarrow R$ it follows for $r$ finite that

$$
\left\{b \in B: \sup _{a \in A} f(a, b) \leq r\right\}=\cap_{a \in A}\{b \in B: f(a, b) \leq r\}
$$

we obtain immediately for $b \longmapsto f(a, b), a \in A$ lower semicontinuous that the function $b \longmapsto \sup _{a \in A} f(a, b)$ is also lower semicontinuous. This implies by Lemma 2 for $B$ a compact topological space and using Lemma 1 that there exists some $b_{0} \in B$ satisfying

$$
\sup _{a \in A} f\left(a, b_{0}\right)=\inf _{b \in B} \sup _{a \in A} f(a, b)=\inf _{b \in B} \sup _{\lambda \in \mathcal{P}_{F}(A)} f_{e}\left(\lambda, \epsilon_{b}\right) .
$$

By a symmetry argument a similar observation holds for player 1 if $A$ is a compact topological space and $a \longmapsto f(a, b)$ is upper semicontinuous for every $b \in B$.

Definition 6. A function $k: X \rightarrow R$ with $X$ a topological space is called inf-compact if all its lower level sets $\{x \in X: k(x) \leq r\}, r \in R$ are compact. It is called sup-compact if all its upper level sets $\{x \in X: k(x) \geq r\}, r \in R$ are compact.

If $B$ is a Hausdorff space it is shown in Chapter 9 of [6] that a compact subset of $B$ is closed. This proves for $B$ Hausdorff that every inf-compact (supcompact) function is actually lower-semicontinuous (upper semicontinuous). Using now Lemma 2 and Definition 6 one can prove the following important result.

Lemma 3. If the pure strategy set $B$ is a topological space and there exist some $I_{0} \in \mathcal{F}(A)$ such that the function $b \longmapsto \max _{a \in I_{0}} f(a, b)$ is inf-compact and $b \longmapsto f(a, b)$ is lower semicontinuous for every $a \in A$, then

$$
\sup _{I \in \mathcal{F}(A)} \inf _{b \in B} \sup _{a \in I} f(a, b)=\inf _{b \in B} \sup _{a \in A} f(a, b) .
$$

Moreover, the inf in the last expression is attained and so, it can be replaced by $\min$.

Proof. Introducing $\beta:=\sup _{I \in \mathcal{F}(A)} \inf _{b \in B} \sup _{a \in I} f(a, b)$ we first verify that

$$
\inf _{b \in B} \sup _{a \in A} f(a, b) \leq \beta+\epsilon
$$

for every $\epsilon>0$. Consider for $\epsilon>0$ the nonempty set

$$
F_{\alpha}(\epsilon):=\left\{b \in B: \max _{a \in I_{0} \cup\{\alpha\}} f(a, b) \leq \beta+\epsilon\right\}, \alpha \in A \backslash I_{0} .
$$


Since the function $b \longmapsto f(a, b)$ is lower semicontinuous for every $a \in A$ it follows by relation (20) that the nonempty set $F_{\alpha}(\epsilon)$ is closed for every $\alpha \in A \backslash I_{0}$. Moreover, for every finite set $I \subseteq A \backslash I_{0}$ we obtain by the definition of $\beta$ that $\cap_{\alpha \in I} F_{\alpha}(\epsilon)$ is nonempty and

$$
F_{\alpha}(\epsilon) \subseteq\left\{b \in B: \max _{a \in I_{0}} f(a, b) \leq \beta+\epsilon\right\}
$$

for any $\alpha \in A \backslash I_{0}$. By assumption the last set in relation (21) is compact and we have shown that the collection $F_{\alpha}(\epsilon), \alpha \in A \backslash I_{0}$ of closed sets satisfies the finite intersection property. This shows that $\cap_{\alpha \in A \backslash I_{0}} F_{\alpha}(\epsilon)$ is nonempty and since

$$
\cap_{\alpha \in A \backslash I_{0}} F_{\alpha}(\epsilon)=\left\{b \in B: \sup _{a \in A} f(a, b) \leq \beta+\epsilon\right\}
$$

we obtain

$$
\inf _{b \in B} \sup _{a \in A} f(a, b) \leq \beta+\epsilon .
$$

Since $\epsilon>0$ is arbitrary, this implies $\inf _{b \in B} \sup _{a \in A} f(a, b)=\beta$ and to show that the infimum is actually attained, we observe the following. Since by relation (22) we obtain for every $\epsilon>0$ that

$$
G(\epsilon):=\cap_{\alpha \in A \backslash I_{0}} F_{\alpha}(\epsilon)
$$

is a closed nonempty set of the compact set $\left\{b \in B: \max _{a \in I_{0}} f(a, b) \leq\right.$ $\beta+\epsilon\}$ the finite intersection property also holds for the decreasing collection $G(\epsilon), \epsilon>0$. This shows that

$$
\inf _{b \in B} \sup _{a \in A} f(a, b)=\min _{b \in B} \sup _{a \in A} f(a, b)
$$

and so the infimum can be replaced by min.

An important special case of Lemma 3 is given by $B$ a compact topological space and $b \longmapsto f(a, b)$ is lower semicontinuous for every $a \in A$. Since every closed subset of a compact set is compact (see Chapter 9 of [6]), it is obvious that the conditions of Lemma 3 are satisfied. A symmetrical version of Lemma 3 needed in the next proof of Wald's minimax theorem is given by

$$
\inf _{J \in \mathcal{F}(B)} \sup _{a \in A} \min _{b \in J} f(a, b)=\max _{a \in A} \inf _{b \in B} f(a, b) .
$$

and this holds if the function $a \longmapsto f(a, b)$ is upper semicontinuous for every $b \in B$ and there exist some $J_{0} \in \mathcal{F}(B)$ such that the function $a \longmapsto \min _{b \in J_{0}} f(a, b)$ is sup-compact. A sufficient condition for this is given by $A$ a compact topological space and $a \longmapsto f(a, b)$ is upper semicontinuous for every $b \in B$. We are now able to give a proof of Wald's minimax result using the strong duality theorem for linear programming and relation (23).

Proof. (Alternative proof of Wald's minimax theorem)

By relation (14) with $A_{0}$ replaced by $B$ it follows for $I$ belonging to $\mathcal{F}(A)$ that 


$$
\inf _{\mu \in \mathcal{P}_{F}(B)} \max _{a \in I} f_{e}\left(\epsilon_{a}, \mu\right)=\inf _{J \in \mathcal{F}(B)} \min _{\mu \in \mathcal{P}(J)} \max _{a \in I} f_{e}\left(\epsilon_{a}, \mu\right) .
$$

For every $J \in \mathcal{F}(B)$ the optimization problem

$$
\min _{\mu \in \mathcal{P}(J)} \max _{a \in I} f_{e}\left(\epsilon_{a}, \mu\right)=\min \left\{z: z \geq f_{e}\left(\epsilon_{a}, \mu\right), a \in I, \mu \in \mathcal{P}(J)\right\}
$$

is a linear programming problem with a finite optimal solution. Hence by the strong duality theorem for linear programming ([22]) we obtain the minimax result given by

$$
\min _{\mu \in \mathcal{P}(J)} \max _{a \in I} f_{e}\left(\epsilon_{a}, \mu\right)=\max _{\lambda \in \mathcal{P}(I)} \min _{b \in J} f_{e}\left(\lambda, \epsilon_{b}\right) .
$$

Applying now relations (24) and (25) yields

$$
\inf _{\mu \in \mathcal{P}_{F}(B)} \max _{a \in I} f_{e}\left(\epsilon_{a}, \mu\right)=\inf _{J \in \mathcal{F}(B)} \max _{\lambda \in \mathcal{P}(I)} \min _{b \in J} f_{e}\left(\lambda, \epsilon_{b}\right) .
$$

Moreover, since the set $I$ is finite and hence $\Delta_{|I|} \subseteq R^{|I|}$ being closed and bounded and hence compact (in the Euclidean topology) and $\lambda \longmapsto f_{e}\left(\lambda, \epsilon_{b}\right)$ is continuous on $\mathcal{P}(I)$ for every $b \in B$ we may use relation (23) with the set $A$ replaced by $\mathcal{P}(I)$ and the function $f(a, b)$ by $f_{e}\left(\lambda, \epsilon_{b}\right)$. This shows

$$
\inf _{J \in \mathcal{F}(B)} \max _{\lambda \in \mathcal{P}(I)} \min _{b \in J} f_{e}\left(\lambda, \epsilon_{b}\right)=\max _{\lambda \in \mathcal{P}(I)} \inf _{b \in B} f_{e}\left(\lambda, \epsilon_{b}\right)
$$

and so we obtain by relation (26) that

$$
\inf _{\mu \in \mathcal{P}_{F}(B)} \max _{a \in I} f_{e}\left(\epsilon_{a}, \mu\right)=\max _{\lambda \in \mathcal{P}(I)} \inf _{b \in B} f_{e}\left(\lambda, \epsilon_{b}\right) .
$$

Finally by Lemma 1 (replace $B_{0}$ by $B$ ) Wald's minimax result is verified.

Actually the minimax result

$$
\min _{\mu \in \mathcal{P}(J)} \max _{a \in I} f_{e}\left(\epsilon_{a}, \mu\right)=\max _{\lambda \in \mathcal{P}(I)} \min _{b \in J} f_{e}\left(\lambda, \epsilon_{b}\right) .
$$

was first proved by von Neumann in 1928 ([15]). In fact in this paper a more general minimax result for a continuous payoff function defined on the Cartesian product of compact simplices which is quasiconvex in $B$ and quasiconcave in $A$ was shown. This result seems to have been forgotten in the literature (the special case in relation (27) was published in [23]) and was later independently generalized by Sion ([14]) in 1958. A useful consequence of Lemma 3 and Wald's minimax result is given by Kneser's minimax result ([24]).

Lemma 4. If the set $A$ is a compact convex subset of a linear topological space, $B$ is a convex subset of a linear space, the payoff function $f: A \times B \rightarrow R$ is affine in both variables and $a \longmapsto f(a, b)$ is upper semicontinuous for every $b \in B$, then

$$
\sup _{a \in A} \inf _{b \in B} f(a, b)=\inf _{b \in B} \sup _{a \in A} f(a, b)
$$

and in both expressions the sup can be replaced by max. 
Proof. Since $A$ is a compact convex topological space and the function $a \longmapsto$ $f(a, b)$ is upper semicontinuous for every $b \in B$ we obtain by relation (23) that

$$
\max _{a \in A} \inf _{b \in B} f(a, b)=\inf _{J \in \mathcal{F}(B)} \max _{a \in A} \min _{b \in J} f(a, b) .
$$

Considering now any $\lambda$ belonging to $\mathcal{P}_{F}(A)$ and $b \in B$ it follows that there exists some finite set $\left\{a_{1}, \ldots, a_{k}\right\} \subseteq A$ and $s(\lambda)^{\top} \in \Delta_{k}$ such that

$$
\lambda=\sum_{i=1}^{k} s_{i}(\lambda) \epsilon_{a_{i}} .
$$

This implies, using $a \longmapsto f(a, b)$ is affine for every $b \in B$ and $A$ is a convex set, that

$$
\max _{a \in A} \min _{b \in J} f(a, b) \geq \min _{b \in J} f\left(\sum_{i=1}^{k} s_{i}(\lambda) a_{i}, b\right)=\min _{b \in J} f_{e}\left(\lambda, \epsilon_{b}\right) .
$$

Since $\lambda \in \mathcal{P}_{F}(A)$ is arbitrary, relation (29) yields

$$
\max _{a \in A} \min _{b \in J} f(a, b) \geq \sup _{\lambda \in \mathcal{P}_{F}(A)} \min _{b \in J} f_{e}\left(\lambda, \epsilon_{b}\right)
$$

and by Lemma 1 and relation (14) with $A_{0}$ replaced by $A$ this implies

$$
\max _{a \in A} \min _{b \in J} f(a, b)=\sup _{\lambda \in \mathcal{P}_{F}(A)} \min _{\mu \in \mathcal{P}(J)} f_{e}(\lambda, \mu) .
$$

Applying now the symmetrical version of Wald's minimax theorem listed in relation (17) to the last part of relation (30) yields

$$
\max _{a \in A} \min _{b \in J} f(a, b)=\min _{\mu \in \mathcal{P}(J)} \sup _{\lambda \in \mathcal{P}_{F}(A)} f_{e}(\lambda, \mu) .
$$

Hence by relations (28), (31), (14) and Lemma 1 we obtain

$$
\max _{a \in A} \inf _{b \in B} f(a, b)=\inf _{\mu \in \mathcal{P}_{F}(B)} \sup _{a \in A} f_{e}\left(\epsilon_{a}, \mu\right) .
$$

Since the function $b \longmapsto f(a, b)$ is affine for every $a \in A$ and the set $B$ is convex we obtain as in the first part of this proof that

$$
\inf _{\mu \in \mathcal{P}_{F}(B)} \sup _{a \in A} f_{e}\left(\epsilon_{a}, \mu\right)=\inf _{b \in B} \sup _{a \in A} f(a, b)=\inf _{b \in B} \max _{a \in A} f(a, b)
$$

and in combination with relation (32) the desired result follows.

Actually one can show that the minimax results of Wald, von Neumann and Kneser can be easily derived from each other. For more equivalent minimax results the reader is referred to ([25]). An easy consequence of Wald's minimax theorem useful in Section 2 is given by the following.

Theorem 3. For any payoff function $f: A \times B \rightarrow R$

$$
\sup _{I \in \mathcal{F}(A)} \inf _{\mu \in \mathcal{P}_{F}(B)} \max _{a \in I} f_{e}\left(\epsilon_{a}, \mu\right)=\sup _{\lambda \in \mathcal{P}_{F}(A)} \inf _{\mu \in \mathcal{P}_{F}(B)} f_{e}(\lambda, \mu) .
$$


Proof. By Lemma 1 and Wald's minimax theorem we obtain for every $I$ belonging to $\mathcal{F}(A)$ that

$$
\inf _{\mu \in \mathcal{P}_{F}(B)} \max _{a \in I} f_{e}\left(\epsilon_{a}, \mu\right)=\max _{\lambda \in \mathcal{P}(I)} \inf _{\mu \in \mathcal{P}_{F}(B)} f_{e}(\lambda, \mu) .
$$

Since by relation (14)

$$
\sup _{I \in \mathcal{F}(A)} \max _{\lambda \in \mathcal{P}(I)} \inf _{\mu \in \mathcal{P}_{F}(B)} f_{e}(\lambda, \mu)=\sup _{\lambda \in \mathcal{P}_{F}(A)} \inf _{\mu \in \mathcal{P}_{F}(B)} f_{e}(\lambda, \mu)
$$

the desired result follows using relation (33).

\section{On necessary and sufficient conditions for minimax theorems}

In this section we will derive necessary and sufficient conditions for the different minimax equalities listed in relations (6) up to (8) by means of the extension of Wald's minimax result listed in Theorem 3. Observe these minimax results are equivalent to the existence of "optimal" strategies for two player zero-sum noncooperative games under different conditions on the use of the strategy sets of the two players. To derive these conditions for relation (6), we introduce the following class of functions.

Definition 7. The payoff function $f: A \times B \rightarrow R$ belongs to the set $\mathcal{U}_{0}$ if

$$
\sup _{I \in \mathcal{F}(A)} \inf _{\mu \in \mathcal{P}_{F}(B)} \max _{a \in I} f_{e}\left(\epsilon_{a}, \mu\right)=\inf _{\mu \in \mathcal{P}_{F}(B)} \sup _{a \in A} f_{e}\left(\epsilon_{a}, \mu\right) .
$$

A game theoretic interpretation of a payoff function $f$ belonging to the set $\mathcal{U}_{0}$ is given by the observation that for player 2 using the mixed strategy set $\mathcal{P}_{F}(B)$ and the minimax approach it does not make any difference whether his opponent given by player 1 selects a pure strategy from the set $A$ or first considers all finite subsets of $A$ and then selects from one of these finite subsets his pure strategy. However, it might be possible that the value for player 2 cannot be achieved if he uses the set $\mathcal{P}_{F}(B)$ of mixed strategies.

Theorem 4. The minimax result in relation (6), given by

$$
\inf _{\mu \in \mathcal{P}_{F}(B)} \sup _{\lambda \in \mathcal{P}_{F}(A)} f_{e}(\lambda, \mu)=\sup _{\lambda \in \mathcal{P}_{F}(A)} \inf _{\mu \in \mathcal{P}_{F}(B)} f_{e}(\lambda, \mu)
$$

holds if and only if the function $f$ belongs to the set $\mathcal{U}_{0}$.

Proof. By Theorem 3 and the definition of $\mathcal{U}_{0}$ the result follows immediately.

The importance of the above theorem is that the minimax equality in relation (6) is replaced by an easier condition. Notice that $\mathcal{U}_{0}$ is automatically satisfied if $A$ is a finite set. In this way Wald's minimax theorem is a direct consequence of Theorem 4. Moreover, we will show at the end of this section 
that a minimax result derived by Ville ([26]) is an easy consequence of Theorem 4 . We do this by showing that the conditions imposed on the payoff function $f$ and the pure strategy sets $A$ and $B$ imply that the function $f$ should belong to the set $\mathcal{U}_{0}$. Actually by a symmetric argument (replace $f$ by $-f$ and reverse the sets $A$ and $B$ !) one can also introduce the following class of functions.

Definition 8. The payoff function $f: A \times B \rightarrow R$ belongs to the set $\mathcal{V}_{0}$ if

$$
\inf _{J \in \mathcal{F}(B)} \sup _{\lambda \in \mathcal{P}_{F}(A)} \min _{b \in J} f_{e}\left(\lambda, \epsilon_{b}\right)=\sup _{\lambda \in \mathcal{P}_{F}(A)} \inf _{b \in B} f_{e}\left(\lambda, \epsilon_{b}\right) .
$$

Using the same symmetry argument the next corollary is an easy consequence of Theorem 4.

Corollary 1. The minimax result in relation (6), given by

$$
\inf _{\mu \in \mathcal{P}_{F}(B)} \sup _{\lambda \in \mathcal{P}_{F}(A)} f_{e}(\lambda, \mu)=\sup _{\lambda \in \mathcal{P}_{F}(A)} \inf _{\mu \in \mathcal{P}_{F}(B)} f_{e}(\lambda, \mu)
$$

holds if and only if the function $f$ belongs to $\mathcal{V}_{0}$.

To derive a necessary and sufficient condition for the minimax equality in relation (7) we introduce the following class of functions.

Definition 9. The function $f: A \times B \rightarrow R$ belongs to the set $\mathcal{U}_{1}$ if

$$
\sup _{I \in \mathcal{F}(A)} \inf _{\mu \in \mathcal{P}_{F}(B)} \max _{a \in I} f_{e}\left(\epsilon_{a}, \mu\right)=\inf _{b \in B} \sup _{a \in A} f(a, b) .
$$

A game theoretic interpretation of the payoff function $f$ belonging to the set $\mathcal{U}_{1}$ is given by the observation that for player 2 using the mixed strategy set $\mathcal{P}_{F}(B)$ and the minimax approach it does not make any difference whether his opponent given by player 1 selects a pure strategy from the set $A$ or first considers all finite subsets of $A$ and then selects from one of these finite subsets his pure strategy. Moreover, the payoff function for player 2 is such that his mixed strategy set is always dominated by his pure strategy set. A sufficient condition for the listed minimax result was discussed in [27].

Theorem 5. The minimax result in relation (7), given by

$$
\inf _{b \in B} \sup _{\lambda \in \mathcal{P}_{F}(A)} f_{e}\left(\lambda, \epsilon_{b}\right)=\sup _{\lambda \in \mathcal{P}_{F}(A)} \inf _{b \in B} f_{e}\left(\lambda, \epsilon_{b}\right) \text {. }
$$

holds if and only if the function $f$ belongs to $\mathcal{U}_{1}$.

Proof. By Lemma 1 the minimax result listed in relation (7) is the same as

$$
\inf _{b \in B} \sup _{a \in A} f(a, b)=\sup _{\lambda \in \mathcal{P}_{F}(A)} \inf _{\mu \in \mathcal{P}_{F}(B)} f_{e}(\lambda, \mu)
$$

Hence by Theorem 3 and the definition of $\mathcal{U}_{1}$ the desired result follows.

Finally we derive a necessary and sufficient condition for the minimax equality listed in relation (8) involving the pure strategy sets $A$ and $B$. 
Definition 10. The function $f: A \times B \rightarrow R$ belongs to the set $\mathcal{U}_{2}$ if

$$
\sup _{\lambda \in \mathcal{P}_{F}(A)} \inf _{b \in B} f_{e}\left(\lambda, \epsilon_{b}\right)=\sup _{a \in A} \inf _{b \in B} f(a, b) .
$$

A game theoretic interpretation of the payoff function $f$ belonging to the set $\mathcal{U}_{2}$ is given by the observation that for player 1 using the mixed strategy set $\mathcal{P}_{F}(A)$ and the minimax approach his mixed strategy set is always dominated by his pure strategy set. This means that player 1 can restrict himself to the set of pure strategies instead of using the set of mixed strategies. One can now show the most well known minimax result.

Theorem 6. The minimax result in relation (8), given by

$$
\inf _{b \in B} \sup _{a \in A} f(a, b)=\sup _{a \in A} \inf _{b \in B} f(a, b) .
$$

holds if and only if the function $f$ belongs to the set $\mathcal{U}_{1} \cap \mathcal{U}_{2}$.

Proof. If the function $f$ belongs to the set $\mathcal{U}_{1} \cap \mathcal{U}_{2}$, then by Lemma 1 (replace $A_{0}$ by $A$ ) and Theorem 5 we obtain

$$
\inf _{b \in B} \sup _{a \in A} f(a, b)=\sup _{\lambda \in \mathcal{P}_{F}(A)} \inf _{b \in B} f_{e}\left(\lambda, \epsilon_{b}\right) .
$$

By the definition of the set $\mathcal{U}_{2}$ this implies that relation (8) holds. To show the reverse implication consider an arbitrary $\lambda$ belonging to $\mathcal{P}_{F}(A)$. By relation (14) there exists some $I_{0} \in \mathcal{F}(A)$ such that $\lambda \in \mathcal{P}\left(I_{0}\right)$ and so we obtain

$$
\inf _{b \in B} f_{e}\left(\lambda, \epsilon_{b}\right) \leq \sup _{I \in \mathcal{F}(A)} \inf _{b \in B} \sup _{a \in I} f(a, b) .
$$

This implies

$$
\sup _{\lambda \in \mathcal{P}_{F}(A)} \inf _{b \in B} f_{e}\left(\lambda, \epsilon_{b}\right) \leq \sup _{I \in \mathcal{F}(A)} \inf _{b \in B} \sup _{a \in I} f(a, b) .
$$

Also by our minimax result listed in relation (8) we obtain

$\sup _{I \in \mathcal{F}(A)} \inf _{b \in B} \sup _{a \in I} f(a, b) \leq \inf _{b \in B} \sup _{a \in A} f(a, b)=\sup _{a \in A} \inf _{b \in B} f(a, b)$

and this shows that

$$
\sup _{\lambda \in \mathcal{P}_{F}(A)} \inf _{b \in B} f_{e}\left(\lambda, \epsilon_{b}\right) \leq \sup _{a \in A} \inf _{b \in B} f(a, b)
$$

Since the reverse inequality trivially holds we can replace the inequality in relation $(56)$ by an equality and so the function $f$ belongs to $\mathcal{U}_{2}$. This implies using again the minimax equality in relation (8) that

$$
\sup _{\lambda \in \mathcal{P}_{F}(A)} \inf _{b \in B} f_{e}\left(\lambda, \epsilon_{b}\right)=\inf _{b \in B} \sup _{a \in A} f(a, b)
$$

and by Theorem 5 the function $f$ belongs to $\mathcal{U}_{1}$.

Again using a symmetry argument (replace $f$ by $-f$ and reverse the sets $A$ and $B$ !) in the definition of the sets $\mathcal{U}_{1}$ and $\mathcal{U}_{2}$ one can introduce the following class of functions. 
Definition 11. The payoff function $f: A \times B \rightarrow R$ belongs to the set $\mathcal{V}_{1}$ if

$$
\inf _{J \in \mathcal{F}(B)} \sup _{\lambda \in \mathcal{P}_{F}(A)} \min _{b \in J} f_{e}\left(\lambda, \epsilon_{b}\right)=\sup _{a \in A} \inf _{b \in B} f(a, b),
$$

while $f: A \times B \rightarrow R$ belongs to the set $\mathcal{V}_{2}$ if

$$
\inf _{\mu \in \mathcal{P}_{F}(B)} \sup _{a \in A} f_{e}\left(\epsilon_{a}, \mu\right)=\inf _{b \in B} \sup _{a \in A} f(a, b) .
$$

By the same symmetry argument one can easily derive the following corollary from Theorem 6 .

Corollary 2. The minimax result in relation (8), given by

$$
\inf _{a \in A} \sup _{b \in B} f(a, b)=\sup _{b \in B} \inf _{a \in A} f(a, b)
$$

holds if and only if $f$ belongs to the set $\mathcal{V}_{1} \cap \mathcal{V}_{2}$.

Before giving a short overview of some minimax theorems which appeared in the literature we list some definitions and results for functions defined on a metric space. Observe we also include the definition of a continuous function on a metric space. Another equivalent definition of a continuous function on a topological space was already given in Definition 5 .

Definition 12. Let $(X, \rho)$ be a metric space with metric $\rho$. The function $k$ : $X \rightarrow R$ is said to be continuous at the point $x \in X$ if for every $\epsilon>0$ there exists some $\delta>0$ such that $|k(x)-k(y)|<\epsilon$ for every $y \in X$ satisfying $\rho(x, y)<\delta$. It is called continuous on $X$ if it is continuous at every point $x \in X$. A function $k: X \rightarrow R$ is called uniformly continuous on $X$ if for every $\epsilon>0$ there exists some $\delta>0$ such that for any $x, y \in X$ satisfying $\rho(x, y)<\delta$ it holds that $|k(x)-k(y)|<\epsilon$. Finally, a collection of functions $k_{\gamma}: X \rightarrow R, \gamma \in \Gamma$ is called equicontinuous if for every $\epsilon>0$ there exists some $\delta>0$ such that for every $x, y \in X$ satisfying $\rho(x, y)<\delta$ it holds that $\left|k_{\gamma}(x)-k_{\gamma}(y)\right|<\epsilon$ for every $\gamma \in \Gamma$.

Recall in a metric space $(X, \rho)$ with metric $\rho$, the open ball $B\left(x_{0}, \delta\right)$ with center $x_{0}$ and radius $\delta>0$ is given by

$$
B\left(x_{0}, \delta\right):=\left\{x \in X: \rho\left(x, x_{0}\right)<\delta\right\} .
$$

We now list the following well-known result ([6], [28]).

Lemma 5. For $(X, \rho)$ a compact metric space with metric $\rho$ a function $k$ : $X \rightarrow R$ continuous on $X$ is uniformly continuous on $X$.

Proof. Let $\epsilon>0$ and consider an arbitrary $x \in X$. Since $k$ is continuous at $x$ there exists some $\delta_{x}>0$ such that $|k(x)-k(y)|<2^{-1} \epsilon$ for every $y$ belonging to $B\left(x, \delta_{x}\right)$. Clearly the collection of open balls $B\left(x, 2^{-1} \delta_{x}\right), x \in X$ is a covering of $X$ and this implies by the compactness of $X$ that there exists some finite set $F=\left\{x_{1}, \ldots, x_{n}\right\} \subseteq X$ satisfying 


$$
X=\cup_{i=1}^{n} B\left(x_{i}, 2^{-1} \delta_{x_{i}}\right) .
$$

Let now $\delta:=4^{-1} \min _{1<i<n} \delta_{x_{i}}$ and consider two points $y, z \in X$ satisfying $\rho(z, y)<\delta$. By relation (36) there exists some $1 \leq i^{*} \leq n$ such that $\rho\left(y, x_{i^{*}}\right)<$ $2^{-1} \delta_{x_{i^{*}}}$ and so $\left|f\left(x_{i^{*}}\right)-f(y)\right| \leq 2^{-1} \epsilon$. By the triangle inequality of a metric we also obtain, using $\rho(z, y)<\delta$, that

$$
\rho\left(z, x_{i^{*}}\right) \leq \rho(z, y)+\rho\left(y, x_{i^{*}}\right)<\delta+2^{-1} \delta_{x_{i^{*}}} \leq \delta_{x_{i}^{*}},
$$

and so $\left|f(z)-f\left(x_{i^{*}}\right)\right|<2^{-1} \epsilon$. This shows that

$$
|f(z)-f(y)| \leq\left|f(z)-f\left(x_{i^{*}}\right)\right|+\left|f\left(x_{i^{*}}\right)-f(y)\right|<2^{-1} \epsilon+2^{-1} \epsilon=\epsilon
$$

and we have shown that the function $k$ is uniformly continuous on $X$.

We now recall the minimax equality listed in relation (6). In 1938 Ville ([26]) proved a generalization of the well-known von Neumann minimax result listed in relation (27). This result is shown in Theorem 7 and serves as an important tool in infinite zero-sum or antagonistic game theory ([3]).

Theorem 7. If $A$ and $B$ are nonempty compact sets in metric spaces and the payoff function $f: A \times B \rightarrow R$ is continuous, then

$$
\sup _{\lambda \in \mathcal{P}_{F}(A)} \inf _{\mu \in \mathcal{P}_{F}(B)} f_{e}(\lambda, \mu)=\inf _{\mu \in \mathcal{P}_{F}(B)} \sup _{\lambda \in \mathcal{P}_{F}(A)} f_{e}(\lambda, \mu) .
$$

To prove Theorem 7 we show that the conditions imposed on $f$ and the sets $A$ and $B$ imply that the function $f$ belongs to the set $\mathcal{U}_{0}$. Applying then Theorem 4 yields a proof of Ville's minimax theorem. Actually we show the following result.

Lemma 6. If the set $A$ is a compact metric space with metric $\rho$ and the collection of functions $f_{b}: A \rightarrow R, b \in B$ given by $f_{b}(a):=f(a, b)$ is equicontinuous with $f$ the payoff function, then $f$ belongs to $\mathcal{U}_{0}$. In particular, if $f$ is continuous and the sets $A$ and $B$ are compact metric spaces, then $f$ belongs to $\mathcal{U}_{0}$.

Proof. For the proof of the first part it is obvious that

$$
\sup _{I \in \mathcal{F}(A)} \inf _{\mu \in \mathcal{P}_{F}(B)} \max _{a \in I} f_{e}\left(\epsilon_{a}, \mu\right) \leq \inf _{\mu \in \mathcal{P}_{F}(B)} \sup _{a \in A} f_{e}\left(\epsilon_{a}, \mu\right) .
$$

To show the result it is therefore sufficient to verify that for every $\epsilon>0$ there exists some set $I_{\epsilon} \in \mathcal{F}(A)$ satisfying

$$
\inf _{\mu \in \mathcal{P}_{F}(B)} \sup _{a \in A} f_{e}\left(\epsilon_{a}, \mu\right) \leq \inf _{\mu \in \mathcal{P}_{F}(B)} \sup _{a \in I_{\epsilon}} f_{e}\left(\epsilon_{a}, \mu\right)+\epsilon .
$$

Let $\epsilon>0$ be given. Since the collection of functions $f_{b}, b \in B$ is equicontinuous one can find some $\delta>0$ such that for every $a_{1}, a_{2} \in A$ satisfying $\rho\left(a_{1}, a_{2}\right)<\delta$ it holds that 


$$
\left|f\left(a_{1}, b\right)-f\left(a_{2}, b\right)\right|<\epsilon
$$

for every $b \in B$. Clearly the collection of open balls $B(a, \delta), a \in A$ covers $A$ and by the compactness of $A$ one can find a finite set $I_{\epsilon} \in \mathcal{F}(A)$ such that

$$
A=\cup_{a \in I_{\epsilon}} B(a, \delta) .
$$

Consider now an arbitrary $\mu \in \mathcal{P}_{F}(B)$. By relation (37) and $f_{b}, b \in B$ equicontinuous it follows for any $a \in A$ that there exists some $a_{0} \in I_{\epsilon}$ such that

$$
\left|f(a, b)-f\left(a_{0}, b\right)\right|<\epsilon
$$

for every $b \in B$. Hence by the definition of $\mathcal{P}_{F}(B)$ this implies

$$
f_{e}\left(\epsilon_{a}, \mu\right) \leq f_{e}\left(\epsilon_{a_{0}}, \mu\right)+\epsilon \leq \sup _{a \in I_{\epsilon}} f_{e}\left(\epsilon_{a}, \mu\right)+\epsilon .
$$

Since $a \in A$ is arbitrary, it follows by relation (38) that

$$
\sup _{a \in A} f_{e}\left(\epsilon_{a}, \mu\right) \leq \sup _{a \in I_{\epsilon}} f_{e}\left(\epsilon_{a}, \mu\right)+\epsilon
$$

and this implies (using $\mu$ is arbitrary) the desired inequality. To verify the second part, it follows by the continuity of the function $f$ on the compact metric space $A \times B$ and Lemma 5 that the function $f$ is uniformly continuous on $A \times B$. This shows that the collection $f_{b}, b \in B$ is equicontinuous and by the first part the desired result follows.

Actually the conditions imposed by Ville can be improved in the following way $([29])$.

Theorem 8. If the pure strategy sets $A$ and $B$ are compact Hausdorff spaces and $b \longmapsto f(a, b)$ is lower semicontinuous for every $a \in A$ and $a \longmapsto f(a, b)$ is upper semicontinuous for every $b \in B$ and the payoff function $f$ belongs to the space of Borel measurable functions which are Lebesgue absolutely integrable with respect to any Borel product probability measure $\mu \otimes \lambda$ on $B \times A$, then

$$
\sup _{\lambda \in \mathcal{P}_{F}(A)} \inf _{\mu \in \mathcal{P}_{F}(B)} f_{e}(\lambda, \mu)=\inf _{\mu \in \mathcal{P}_{F}(B)} \sup _{\lambda \in \mathcal{P}_{F}(A)} f_{e}(\lambda, \mu) .
$$

Again this result (for an alternative proof see [29]) can be verified by showing that the above conditions imply that the function $f$ belongs to $\mathcal{U}_{0}$. Since its proof involves classical results from the set of Borel measures on a compact Hausdorff space ([8], [9]) and these results are beyond the scope of this chapter, we refer the reader to [29] for more details on the used techniques. We also like to mention for $\mathcal{P}(A)(\mathcal{P}(B))$ denoting the set of Borel probability measures on $A(B)$ that under the conditions of the next lemma one can show by a similar type of proof as in Lemma 1 that

$$
\sup _{\lambda \in \mathcal{P}(A)} f_{e}(\lambda, \mu)=\sup _{a \in A} f_{e}\left(\epsilon_{a}, \mu\right)
$$

for every $\mu \in \mathcal{P}(B)$ and

$$
\inf _{\mu \in \mathcal{P}(B)} f_{e}(\lambda, \mu)=\inf _{b \in B} f_{e}\left(\lambda, \epsilon_{b}\right)
$$

for every $\lambda \in \mathcal{P}(A)$. 
Lemma 7. If the pure strategy set $A$ and $B$ are compact Hausdorff spaces and the function $b \longmapsto f(a, b)$ is lower semicontinuous for every $b \in B$ and $a \longmapsto f(a, b)$ is upper semicontinuous for every $a \in A$ and $f$ belongs to the space of Borel measurable functions which are Lebesgue absolutely integrable with respect to any Borel product probability measure $\mu \otimes \lambda$ on $B$, then the function $f$ belongs to $\mathcal{U}_{0}$.

Proof. Since the function $b \longmapsto f(a, b)$ is Lebesgue absolutely integrable for any Borel probability measure $\mu$ on the set $B$, one can show (see Corollary 2.2 of [30] that

$$
\inf _{\mu \in \mathcal{P}_{F}(B)} \max _{a \in I} f_{e}\left(\epsilon_{a}, \mu\right)=\inf _{\mu \in \mathcal{P}(B)} \sup _{a \in I} f_{e}\left(\epsilon_{a}, \mu\right) .
$$

for any $I \in \mathcal{F}(A)$. Hence we obtain

$\sup _{I \in \mathcal{F}(A)} \inf _{\mu \in \mathcal{P}_{F}(B)} \max _{a \in I} f_{e}\left(\epsilon_{a}, \mu\right)=\sup _{I \in \mathcal{F}(A)} \inf _{\mu \in \mathcal{P}(B)} \sup _{a \in I} f_{e}\left(\epsilon_{a}, \mu\right)$.

In the remainder of the proof we will now verify that

$$
\sup _{I \in \mathcal{F}(A)} \inf _{\mu \in \mathcal{P}(B)} \sup _{a \in I} f_{e}\left(\epsilon_{a}, \mu\right) \geq \inf _{\mu \in \mathcal{P}_{F}(B)} \sup _{a \in A} f_{e}\left(\epsilon_{a}, \mu\right) .
$$

Assuming for the moment that this holds it follows by (41) that $f$ belongs to $\mathcal{U}_{0}$. To prove relation (42) we observe for $B$ a compact Hausdorff space that the set $\mathcal{P}(B)$ is compact in the weak* topology ([8], [9]) and the function $\mu \longmapsto f_{e}\left(\epsilon_{a}, \mu\right)$ is lower semicontinuous with respect to the weak* topology (see Lemma 12 of [29]). Hence by Lemma 3 (replace $B$ by $\mathcal{P}(B)$ and $f(a, b)$ by $\left.f_{e}\left(\epsilon_{a}, \mu\right)\right)$ and relation (39) it follows that

$$
\sup _{I \in \mathcal{F}(A)} \inf _{\mu \in \mathcal{P}(B)} \sup _{a \in I} f_{e}\left(\epsilon_{a}, \mu\right)=\inf _{\mu \in \mathcal{P}(B)} \sup _{\lambda \in \mathcal{P}(A)} f_{e}(\lambda, \mu) .
$$

Again by Lemma 12 of [29] the function $\mu \longmapsto f_{e}(\lambda, \mu)$ is upper semicontinuous and since $\mathcal{P}(A)$ is also weak ${ }^{*}$ compact we obtain by Kneser's minimax theorem (Lemma 4) (replace $A$ by $\mathcal{P}(A)$ and $f(a, b)$ by the biaffine function $f_{e}(\lambda, \mu)$ ) and relation (40) that

$$
\inf _{\mu \in \mathcal{P}(B)} \sup _{\lambda \in \mathcal{P}(A)} f_{e}(\lambda, \mu)=\sup _{\lambda \in \mathcal{P}(A)} \inf _{b \in B} f_{e}\left(\lambda, \epsilon_{b}\right) .
$$

Again by the weak ${ }^{*}$ compactness of $\mathcal{P}(A)$ and relation (40) it follows that

$$
\sup _{\lambda \in \mathcal{P}(A)} \inf _{b \in B} f_{e}\left(\lambda, \epsilon_{b}\right)=\inf _{J \in \mathcal{F}(B)} \sup _{\lambda \in \mathcal{P}(A)} \inf _{\mu \in \mathcal{P}(J)} f_{e}(\lambda, \mu) .
$$

It is now obvious that

$$
\begin{aligned}
& \inf _{J \in \mathcal{F}(B)} \sup _{\lambda \in \mathcal{P}(A)} \inf _{\mu \in \mathcal{P}(J)} f_{e}(\lambda, \mu) \geq \\
& \inf _{J \in \mathcal{F}(B)} \sup _{\lambda \in \mathcal{P}_{F}(A)} \inf _{\mu \in \mathcal{P}(J)} f_{e}(\lambda, \mu)
\end{aligned}
$$


and by Wald's minimax theorem and Lemma 1

$$
\inf _{J \in \mathcal{F}(B)} \sup _{\lambda \in \mathcal{P}_{F}(A)} \inf _{\mu \in \mathcal{P}(J)} f_{e}(\lambda, \mu)=\inf _{\mu \in \mathcal{P}_{F}(B)} \sup _{a \in A} f_{e}\left(\epsilon_{a}, \mu\right) .
$$

This implies by relations (43) up to (46) that

$$
\sup _{I \in \mathcal{F}(A)} \inf _{\mu \in \mathcal{P}(B)} \sup _{a \in I} f_{e}\left(\epsilon_{a}, \mu\right) \geq \inf _{\mu \in \mathcal{P}_{F}(B)} \sup _{a \in A} f_{e}\left(\epsilon_{a}, \mu\right)
$$

and so relation (42) is proved.

We will now consider the minimax equality listed in relation (7) and introduce the following definition used in ([27]).

Definition 13. The payoff function $f: A \times B \rightarrow R$ is called weakly convexlike on $B$ (or belongs to the set $W C_{B}$ ) if for every finite set $I \subseteq A$

$$
\inf _{\alpha \in \Delta_{n}, b_{i} \in B, 1 \leq i \leq n, n \in N} \max _{a \in I} \sum_{i=1}^{n} \alpha_{i} f\left(a, b_{i}\right) \geq \inf _{b \in B} \max _{a \in I} f(a, b) .
$$

An alternative representation of the above definition is given by

$$
\inf _{\mu \in \mathcal{P}_{F}(B)} \max _{a \in I} f_{e}\left(\epsilon_{a}, \mu\right) \geq \inf _{b \in B} \max _{a \in I} f(a, b)
$$

for every $I$ belonging to $\mathcal{F}(A)$. Since the set $B$ can be identified with the set $\left(\epsilon_{b}\right)_{b \in B}$ it follows for $I \in \mathcal{F}(A)$ that

$$
\inf _{\mu \in \mathcal{P}_{F}(B)} \max _{a \in I} f_{e}\left(\epsilon_{a}, \mu\right) \leq \inf _{b \in B} \max _{a \in I} f(a, b)
$$

and this shows that in Definition 13 the inequality for a weakly convexlike function on $B$ can be replaced by an equality. Again a function belonging to $W C_{B}$ has a clear game theoretical interpretation: for any finite set of pure strategies of player 1 it follows that player 2 using its mixed strategy set $\mathcal{P}_{F}(B)$ can restrict himself to its set of pure strategies. The next result is proved in $([27])$.

Theorem 9. If $B$ is a compact topological space, the payoff function $f$ is weakly convexlike on $B$ and $b \longmapsto f(a, b)$ is lower semicontinuous on $B$ for every $a \in A$, then

$$
\inf _{b \in B} \sup _{\lambda \in \mathcal{P}_{F}(A)} f_{e}\left(\lambda, \epsilon_{b}\right)=\sup _{\lambda \in \mathcal{P}_{F}(A)} \inf _{b \in B} f_{e}\left(\lambda, \epsilon_{b}\right) .
$$

As before, we check that any function satisfying the assumptions above belongs to the set $\mathcal{U}_{1}$, and so by Theorem 5 the minimax result in Theorem 9 is proved.

Lemma 8. If $B$ is a compact topological space and the payoff function $f$ is weakly convexlike on $B$ and $b \longmapsto f(a, b)$ is lower semicontinuous for every $a \in A$, then $f$ belongs to $\mathcal{U}_{1}$. 
Proof. Since the function $f$ is weakly convexlike on $B$ it follows that

$$
\sup _{I \in \mathcal{F}(A)} \inf _{\mu \in \mathcal{P}_{F}(B)} \max _{a \in I} f_{e}\left(\epsilon_{a}, \mu\right)=\sup _{I \in \mathcal{F}(A)} \inf _{b \in B} \max _{a \in I} f(a, b)
$$

By the compactness of the set $B$ and $b \longmapsto f(a, b)$ is lower semicontinuous for every $a \in A$ we may apply Lemma 3 and this shows by the previous equality that $f$ belongs to $\mathcal{U}_{1}$.

Actually as shown by the following counterexample the set of weakly convexlike functions on $B$ with $B$ a compact set and $b \longmapsto f(a, b)$ continuous for every $a \in A$ is strictly included in the set $\mathcal{U}_{1}$. Observe the function $1_{S}$ denotes the characteristic function of the set $S$, i.e., $1_{S}(s)=1$ for $s \in S$ and $1_{S}(s)=0$ otherwise.

Example 2. Let $B=[0,1]$ and $A=\{1,2,3\}$ and introduce the continuous functions $b \longmapsto f(a, b), a \in A$ given by

$$
f(1, b)=2 b 1_{\left\{b \leq 2^{-1}\right\}}+1_{\left\{2^{-1}<b \leq 1\right\}}, f(2, b)=1_{\left\{b \leq 2^{-1}\right\}}+(2-2 b) 1_{\left\{2^{-1}<b \leq 1\right\}}
$$

and $f(3, b)=1_{\{0 \leq b \leq 1\}}$. Since $A$ is a finite set it follows

$$
\sup _{I \in \mathcal{F}(A)} \inf _{\mu \in \mathcal{P}_{F}(B)} \max _{a \in I} f_{e}\left(\epsilon_{a}, \mu\right)=\inf _{\mu \in \mathcal{P}_{F}(B)} \max _{a \in A} f_{e}\left(\epsilon_{a}, \mu\right) .
$$

Using $f(3, b)=1$ for every $b$ we obtain $f_{e}\left(\epsilon_{3}, \mu\right)=1$ for every $\mu \in \mathcal{P}_{F}(B)$ and SO

$$
\inf _{\mu \in \mathcal{P}_{F}(B)} \max _{a \in A} f_{e}\left(\epsilon_{a}, \mu\right)=1 \text {. }
$$

At the same time it is easy to see that $\inf _{b \in B} \max _{a \in A} f(a, b)=1$ and this shows that the function $f$ belongs to $\mathcal{U}_{1}$. Introducing now the set $I_{0}=\{1,2\} \subseteq$ $A$ it follows that

$$
\inf _{b \in B} \max _{a \in I_{0}} f(a, b)=1
$$

Moreover, since $\mu_{0}=2^{-1} \epsilon_{0}+2^{-1} \epsilon_{1}$ belongs to $\mathcal{P}_{F}(B)$ we obtain

$$
\inf _{\mu \in \mathcal{P}_{F}(B)} \max _{a \in I_{0}} f_{e}\left(\epsilon_{a}, \mu\right) \leq \max _{a \in I_{0}} f_{e}\left(\epsilon_{a}, \mu_{0}\right)=2^{-1}
$$

and so $f$ is not weakly convexlike on $B$.

We will now give an overview of the most important different payoff functions $f$ considered in the literature which were used to verify the minimax equality in relation (8). For a more extensive overview the reader should consult [25] or [31]. In a paper by Ky Fan in 1953 ([32]) the following definition is introduced. In the literature these functions are also called convexlike or concavelike.

Definition 14. The payoff function $f: A \times B \rightarrow R$ is called Ky Fan convex on $B$ (or belongs to the set $K F C_{B}$ ) if for every $b_{1}, b_{2} \in B$ and $0<\alpha<1$ there exists some $b_{0} \in B$ satisfying 


$$
f\left(a, b_{0}\right) \leq \alpha f\left(a, b_{1}\right)+(1-\alpha) f\left(a, b_{2}\right)
$$

for every $a \in A$. It is called Ky Fan concave on $A$ or (belongs to the set $K F C_{A}$ ) if for every $a_{1}, a_{2} \in A$ and $0<\alpha<1$ there exists some $a_{0} \in A$ satisfying

$$
f\left(a_{0}, b\right) \geq \alpha f\left(a_{1}, b\right)+(1-\alpha) f\left(a_{2}, b\right)
$$

for every $b \in B$. The payoff function $f: A \times B \rightarrow R$ is called Ky Fan concaveconvex on the Cartesian product $A \times B$, if $f$ is Ky Fan concave on $A$ and $K y$ Fan convex on $B$.

To rewrite the definition of a Ky Fan convex (concave) function in our notation we introduce for $D$ some topological space the set $\mathcal{P}_{2}(D) \subseteq \mathcal{P}_{F}(D)$ of two-point probability measures on $D$. This means that the probability measure $\lambda$ belongs to $\mathcal{P}_{2}(D)$ if and only if

$$
\lambda=s\left(\lambda_{1}\right) \epsilon_{d_{1}}+s\left(\lambda_{2}\right) \epsilon_{d_{2}}
$$

with $d_{i}, 1 \leq i \leq 2$ different elements of the pure strategy set $D$ and $s(\lambda)^{\top}=$ $\left(s\left(\lambda_{1}\right), s\left(\lambda_{2}\right)\right) \in \Delta_{2}$ with $s\left(\lambda_{i}\right)>0,1 \leq i \leq 2$. Using this notation it follows that the payoff function $f: A \times B \rightarrow R$ is Ky Fan convex on $B$ if for every $\mu$ belonging $\mathcal{P}_{2}(B)$ there exists some $b_{0} \in B$ satisfying

$$
f\left(a, b_{0}\right) \leq f_{e}\left(\epsilon_{a}, \mu\right)
$$

for every $a \in A$. Clearly this property also has a clear game theoretical interpretation. For such a payoff function every two-point mixed strategy of player 2 is dominated by a pure strategy. Actually by an easy induction argument one can also show for $f$ Ky Fan convex on $B$ that for any $\mu \in \mathcal{P}_{F}(B)$ there exists some $b_{0} \in B$ satisfying

$$
f\left(a, b_{0}\right) \leq f_{e}\left(\epsilon_{a}, \mu\right)
$$

for any $b \in B$. This means that every mixed strategy of player 2 is dominated by a pure strategy. In [32] the following minimax result is shown.

Theorem 10. If $B$ is compact topological space, the payoff function $f$ is Ky Fan concave-convex on $A \times B$ and $b \longmapsto f(a, b)$ is lower semicontinuous for every $a \in A$, then

$$
\inf _{b \in B} \sup _{a \in A} f(a, b)=\sup _{a \in A} \inf _{b \in B} f(a, b)
$$

and inf can be replaced by min in the above expression.

By the well known symmetry argument (replace $f$ by $-f$ and reverse $A$ and $B$ ) one can easily derive from Theorem 10 that the above minimax result holds if $A$ is a compact topological space, the function $f$ is Ky-Fan concaveconvex on $A \times B$ and $a \longmapsto f(a, b)$ is upper semicontinuous for every $b \in B$. Another more general class of functions was introduced by König in 1968 ([33]). Actually König only introduced the next class with $\beta=\frac{1}{2}$, but indicates at the the end of his paper that the same results also holds with $0<\beta<1$. 
Definition 15. The payoff function $f: A \times B \rightarrow R$ is called König convex on $B$ (or belongs to the set $K C_{B}$ ) if there exists some $0<\beta<1$ such that for every $b_{0}, b_{1} \in B$ there exists some $b_{0} \in B$ satisfying

$$
f\left(a, b_{0}\right) \leq \beta f\left(a, b_{1}\right)+(1-\beta) f\left(a, b_{0}\right)
$$

for every $a \in A$. It is called König concave on $A$ (or belongs to the set $K C_{A}$ ) if there exists some $0<\beta<1$ such that for every $a_{1}, a_{2} \in A$ there exists some $a_{0} \in A$ satisfying

$$
f\left(a_{0}, b\right) \geq \beta f\left(a_{1}, b\right)+(1-\beta) f\left(a_{2}, b\right)
$$

for every $b \in B$. The payoff function $f: A \times B \rightarrow R$ is called König concaveconvex on $A \times B$, if $f$ is König concave on $A$ and König convex on $B$.

Although the above definition is rather technical it has a clear interpretation in game theory. Denoting by $\mathcal{P}_{2, \beta}(D) \subseteq \mathcal{P}_{2}(D)$ the set of two point probability measures on the topological space $D$ with probabilities $\beta$ and $1-\beta$ ( $\beta$ fixed), it means that any mixed strategy of player 2 belonging to $\mathcal{P}_{2, \beta}(B)$ is dominated by a pure strategy. In [33] the same minimax result is shown as in Theorem 10 under the weaker conditions that $B$ is a compact topological space, $b \longmapsto f(a, b)$ is lower semicontinuous for every $a \in A$ and $f$ is König concave-convex on $A \times B$. Another more general class of functions is considered in [34] or [35].

Definition 16. The payoff function $f: A \times B \rightarrow R$ is called closely convex on $B$ (or belongs to the set $C C_{B}$ ) if for every $\epsilon>0,0<\alpha<1$ and $b_{1}, b_{2} \in B$ there exists some $b_{0} \in B$ satisfying

$$
f\left(a, b_{0}\right) \leq \alpha f\left(a, b_{1}\right)+(1-\alpha) f\left(a, b_{2}\right)+\epsilon
$$

for every $a \in A$. It is called closely concave on $A$ (or belongs to the set $C C_{A}$ ) if for every $\epsilon>0,0<\alpha<1$ and $a_{1}, a_{2} \in A$ there exists some $a_{0} \in B$ satisfying

$$
f\left(a_{0}, b\right) \geq \alpha f\left(a_{1}, b\right)+(1-\alpha) f\left(a_{2}, b\right)-\epsilon
$$

for every $b \in B$. The payoff function $f: A \times B \rightarrow R$ is called closely concaveclosely convex on $A \times B$, if $f$ is closely concave on $A$ and closely convex on $B$.

Again in our notation it follows that the payoff function $f$ is closely convex on $B$ if for every $\epsilon>0$ and every $\mu \in \mathcal{P}_{2}(B)$ there exists some $b_{0} \in B$ satisfying

$$
f\left(a, b_{0}\right) \leq f_{e}\left(\epsilon_{a}, \mu\right)+\epsilon
$$

for every $a \in A$. This also has an obvious game theoretical interpretation. In [34] one also shows the minimax result in relation (8) under the weaker 
condition that $B$ is a compact topological space, $b \longmapsto f(a, b)$ is lower semicontinuous for every $a \in A$ and $f$ is closely concave-closely convex on $A \times B$. To show the above results by means of Theorem 6 we need to verify that all the considered payoff functions actually belong to the set $\mathcal{U}_{1} \cap \mathcal{U}_{2}$. In the next result we say that $0 \leq \beta \leq 1$ is a König concave constant on $A$ if for every $\lambda \in \mathcal{P}_{2, \beta}(A)$ there exists some $a_{0} \in A$ satisfying $f\left(a_{0}, b\right) \geq f_{e}\left(\lambda, \epsilon_{b}\right)$ for every $b \in B$.

Lemma 9. It holds that $K F C_{A} \subseteq K C_{A} \subseteq C C_{A} \subseteq \mathcal{U}_{2}$

Proof. It is obvious that the inclusion $K F C_{A} \subseteq K C_{A}$ holds. To show that $K C_{A} \subseteq C C_{A}$ it is sufficient to verify that the set $S \subseteq[0,1]$ given by $S:=$ $\{0 \leq \beta \leq 1: \beta$ a a König concave constant $\}$ satisfies $\operatorname{cl}(S)=[0,1]$. Clearly the numbers 0 and 1 belong to $S$. Since the function $f$ is König concave on $A$ we know that there exists some $0<\beta<1$ belonging to $S$. Moreover, if the numbers $\beta_{i}, i=1,2$ belong to $S$ it follows for every $\lambda_{i}=\beta_{i} \epsilon_{a_{1}}+\left(1-\beta_{i}\right) \epsilon_{a_{2}}$ $\in \mathcal{P}_{2, \beta_{i}}(A)$ with $a_{i} \in A, i=1,2$ that there exists some elements $a\left(\beta_{i}\right) \in A, i=$ 1,2 satisfying

$$
f\left(a\left(\beta_{i}\right), b\right) \geq f_{e}\left(\lambda_{i}, \epsilon_{b}\right)
$$

for every $b \in A$ and $i=1,2$. This implies using $\beta$ belongs to $S$ that for $\lambda=\beta \epsilon_{a\left(\beta_{1}\right)}+(1-\beta) \epsilon_{a\left(\beta_{2}\right)} \in \mathcal{P}_{2, \beta}(A)$ there exists some $a_{0} \in A$ satisfying

$$
f\left(a_{0}, b\right) \geq f_{e}\left(\lambda, \epsilon_{b}\right)
$$

for every $b \in B$. Hence by relation (47) we obtain

$$
f\left(a_{0}, b\right) \geq\left(\beta \beta_{1}+(1-\beta) \beta_{2}\right) f\left(a_{1}, b\right)+\left(1-\beta \beta_{1}-(1-\beta) \beta_{2}\right) f\left(a_{2}, b\right)
$$

for every $b \in B$. This means for any $\beta_{i} \in S, i=1,2$ that also $\beta \beta_{1}+(1-\beta) \beta_{2}$ belongs to $S$ and in [36] it is shown that such a set is dense in $[0,1]$. To verify the last inclusion one can show by induction that for $f$ closely concave on $A$ it follows for every $\epsilon>0$ and $\lambda \in \mathcal{P}_{F}(A)$ that there exists some $a_{0}$ satisfying

$$
f\left(a_{0}, b\right) \geq f_{e}\left(\lambda, \epsilon_{b}\right)-\epsilon
$$

for every $b \in B$. This implies for every $\epsilon>0$ and $\lambda \in \mathcal{P}_{F}(A)$ that

$$
\inf _{b \in B} f\left(a_{0}, b\right) \geq \inf _{b \in B} f_{e}\left(\lambda, \epsilon_{b}\right)-\epsilon
$$

and hence

$$
\sup _{a \in A} \inf _{b \in B} f(a, b) \geq \inf _{b \in B} f_{e}\left(\lambda, \epsilon_{b}\right)-\epsilon .
$$

Since $\lambda \in \mathcal{P}_{F}(A)$ and $\epsilon$ are arbitrary we obtain

$$
\sup _{a \in A} \inf _{b \in B} f(a, b) \geq \sup _{\lambda \in \mathcal{P}_{F}(A)} \inf _{b \in B} f_{e}\left(\lambda, \epsilon_{b}\right),
$$

and so $f$ belongs to $\mathcal{U}_{2}$. 
Actually one can show that the above inclusions are strict ([34]). Moreover, one can also show the following result

Lemma 10. If $B$ is a compact topological space and the function $b \longmapsto f(a, b)$ is lower semicontinuous for every $a \in A$, then $K F C_{B}=K C_{B}=C C_{B} \subseteq \mathcal{U}_{1}$.

Proof. As in Lemma 9 one can show without any additional conditions that $K F C_{B} \subseteq K C_{B} \subseteq C C_{B}$ and to prove equality it is sufficient to verify for $B$ a compact topological space and $b \longmapsto f(a, b)$ lower semicontinuous for every $a \in A$ that $C C_{B} \subseteq K F C_{B}$. We only give a proof of this result for $B$ a compact metric space. (For $B$ a compact topological space one can apply a similar proof replacing sequences by nets (see section 4 of [8]). If the function $f$ is closely convex on the compact metric space $B$, then for every $n \in N, 0<\alpha<1$ and $b_{1}, b_{2} \in B$ there exists some $b_{0, n} \in B$ satisfying

$$
f\left(a, b_{0, n}\right) \leq \alpha f\left(a, b_{1}\right)+(1-\alpha) f\left(a, b_{2}\right)+\frac{1}{n}
$$

for every $a \in A$. Since $B$ is a compact metric space there exists some converging subsequence $b_{0, n}, n \in K \subseteq N$ with limit $b_{0} \in N$. This implies by the lower semicontinuity of the function $b \longmapsto f(a, b)$ for every $a \in A$ that

$$
f\left(a, b_{0}\right) \leq \liminf _{n \in K, n \uparrow \infty} f\left(a, b_{0, n}\right) \leq \alpha f\left(a, b_{1}\right)+(1-\alpha) f\left(a, b_{2}\right)
$$

and so the function $f$ is Ky Fan convex on $B$. To show the inclusion $C C_{B} \subseteq \mathcal{U}_{1}$ we can verify in a similar way as done in the last part of the proof of Lemma 9 that for $f$ closely convex on $B$ it follows for every $I \in \mathcal{F}(A)$ that

$$
\inf _{\mu \in \mathcal{P}_{F}(B)} \max _{a \in I} f_{e}\left(\epsilon_{a}, \mu\right)=\inf _{b \in B} \max _{a \in I} f(a, b)
$$

This implies

$$
\sup _{I \in \mathcal{F}(A)} \inf _{\mu \in \mathcal{P}_{F}(B)} \max _{a \in I} f_{e}\left(\epsilon_{a}, \mu\right)=\sup _{I \in \mathcal{F}(A)} \inf _{b \in B} \max _{a \in I} f(a, b)
$$

and applying Lemma 3 to the last expression in relation (51) we obtain that $f$ belongs to $\mathcal{U}_{1}$.

Using now Lemma 9 and 10 we obtain for $B$ a compact topological space, $b \longmapsto f(a, b)$ is lower semicontinuous for every $a \in A$ and $f$ closely concaveclosely convex on $A \times B$ that $f$ belongs to the set $\mathcal{U}_{1} \cap \mathcal{U}_{2}$ and so by Theorem 6 the classical minimax result in relation (8) holds.

\section{Relations between the different minimax theorems.}

In this section we investigate in more detail the relations between the different minimax results discussed in Section 3 and given by relations (6) up to (8). 
Introducing the notation $L_{i}$ and $R_{i}$ for the left and right-hand side of relation (i) for $i=6,7,8$, we obviously obtain that

$$
L_{8}=L_{7} \geq L_{6} \geq R_{6}=R_{7} \geq R_{8} .
$$

This implies that

$$
(8) \Rightarrow(7) \Rightarrow(6)
$$

Below we show by means of some counterexamples that none of the arrows in relation (53) can be reversed. In the first counterexample we show an instance for which (7) holds and (8) does not hold.

Example 3. Let $A=[0,1] \subset R, B=\left\{b_{1}, b_{2}, b_{3}\right\} \subset R$ and introduce the function $f: A \times B \rightarrow R$ given by

$$
f(a, b)=\left\{\begin{array}{cl}
a^{2} & \text { if } b=b_{1} \\
(a-1)^{2} & \text { if } b=b_{2} \\
2^{-1} & \text { if } b=b_{3}
\end{array}\right.
$$

For this bifunction we have

$$
L_{8}:=\min _{b \in B} \sup _{a \in A} f(a, b)=1 / 2,
$$

while

$$
R_{8}:=\sup _{a \in A} \min _{b \in B} f(a, b)=1 / 4,
$$

and so (8) does not hold. Since $L_{8}=L_{7}=2^{-1}$ and it is obvious to check that $R_{7}=2^{-1}$, we obtain that (7) holds.

In the next counterexample we show an instance for which (6) holds and (7) does not hold.

Example 4. Take $A=[0,1], B=\left\{b_{1}, b_{2}\right\} \subset R$ and introduce the function $f: A \times B \rightarrow R$ given by

$$
f(a, b)=\left\{\begin{array}{cc}
a^{2} & \text { if } b=b_{1} \\
(a-1)^{2} & \text { if } b=b_{2}
\end{array}\right.
$$

Consider now the mixed strategy $\lambda^{*} \in \mathcal{P}_{F}(A)$ given by $\lambda^{*}=2^{-1} \epsilon_{a_{1}}+2^{-1} \epsilon_{a_{2}}$ with $a_{1}=0$ and $a_{2}=1$. It is easy to check that

$$
\min _{b \in B} f_{e}\left(\lambda^{*}, \epsilon_{b}\right)=2^{-1}
$$

and so it follows that $R_{7} \geq 2^{-1}$. Moreover, we observe by the definition of the sets $A$ and $B$ that

$$
L_{6}=\inf _{0 \leq s_{1}(\mu) \leq 1} \sup _{a \in A}\left\{s_{1}(\mu) f\left(a, b_{1}\right)+\left(1-s_{1}(\mu)\right) f\left(a, b_{2}\right)\right\} .
$$

Using now that the last expression in relation (54) equals 


$$
\inf _{0 \leq s_{1}(\mu) \leq 1} \max \left\{s_{1}(\mu), 1-s_{1}(\mu)\right\}=2^{-1}
$$

we obtain that $L_{6}=2^{-1}$. Since we already know that $L_{6} \geq R_{7}=R_{6}$ and $R_{7} \geq 2^{-1}$ we obtain

$$
L_{6}=R_{7}=R_{6}=2^{-1},
$$

It is now easy to check that $L_{7}=1$ and hence we have found an instance for which (6) holds and (7) does not hold.

To conclude these investigations, we give an instance which shows that (6) can also fail. Consider the set $c_{0}$ of all (real valued) sequences converging to 0 . It is well-known that the space $c_{0}$ endowed with the norm

$$
\|a\|_{c_{0}}=\sup _{k \in N}\left|a_{k}\right|
$$

is a Banach space.

Example 5. Let $A=\left\{a=\left(a_{k}\right) \in c_{0}: a_{1}=0\right\}, B=[0,1] \subset R$ and take the function $f: A \times B \rightarrow R$ given by

$$
f(a, b)=f\left(\left(a_{k}\right), b\right)=\left\{\begin{array}{l}
1 \text { if there exist some } k \in N \text { such that } b=a_{k} \\
0 \text { otherwise }
\end{array}\right.
$$

Consider some $\lambda \in \mathcal{P}_{F}(A)$. Hence there exists a finite number of sequences $a^{i}=\left(a_{k}^{i}\right)_{k \in N}, 1 \leq i \leq m$, belonging to $A$ and some vector $s(\lambda)=$ $\left(s_{1}(\lambda), \ldots, s_{m}(\lambda)\right), s_{i}(\lambda)>0$ and $\sum_{i=1}^{m} s_{i}(\lambda)=1$ such that

$$
\lambda=\sum_{i=1}^{m} s_{i}(\lambda) \epsilon_{a^{i}} .
$$

Since the set $[0,1]$ contains more than a countable number of elements one can now choose a number $b \in[0,1]$ such that none of the above sequences $a^{i}, 1 \leq i \leq m$, contain this number. Using this number and the definition of $f$ it can be easily seen that

$$
\inf _{b \in[0,1]} f_{e}\left(\lambda, \epsilon_{b}\right)=\inf _{b \in[0,1]} \sum_{i=1}^{m} s_{i}(\lambda) f\left(a^{i}, b\right)=0,
$$

and so $R_{6}=0$. On the other hand, consider some $\mu \in \mathcal{P}_{F}(B)$. By definition one can find some finite set $\left\{b_{1}, \ldots b_{p}\right\} \subseteq[0,1]$ and a vector $s(\mu)=\left(s_{1}(\mu), \ldots, s_{p}(\mu)\right)$, $s_{j}(\mu)>0$ with $\sum_{j=1}^{p} s_{j}(\mu)=1$ such that

$$
\mu=\sum_{j=1}^{p} s_{j}(\mu) \epsilon_{b_{j}} .
$$

Taking the element $a_{0}:=\left(0, b_{1}, \ldots, b_{p}, 0,0, \ldots\right) \in c_{0}$ it is obvious by the definition of $f$ that

$$
\sup _{a \in A} f_{e}\left(\epsilon_{a}, \mu\right) \geq \sum_{j=1}^{p} s_{j}(\mu) f\left(a_{0}, b_{j}\right)=1 .
$$

Since $f$ is bounded by 1 this shows that

$$
L_{6}:=\inf _{\mu \in \mathcal{P}_{F}(B)} \sup _{a \in A} f_{e}\left(\epsilon_{a}, \mu\right)=1,
$$

and so we have verified that (6) does not hold. 


\section{On Sion's minimax theorem}

In this section we give an alternative and elementary proof of Sion's minimax theorem. This famous result is a generalization of von Neumann's minimax theorem ([15]). Its original proof made use of the KKM lemma which is equivalent to Brouwer's fixed point theorem ([37], [38]). However, as will turn out we do not need such a heavy machinery to verify this result. Actually we will give a proof of a slightly more general result by using a less known technique called the level set method originally developed by Joo ([17]). It remains an open question whether it is possible to verify this minimax result by means of Theorem 6 .

Definition 17. A real valued function $k: C \longrightarrow R$ is called quasiconvex on the (convex) set $C$ if all its lower level sets $\{x \in C: k(x) \leq r\}, r \in R$ are convex. It is called quasiconcave on $C$ if $-f$ is quasiconvex on $C$.

It is well known ([39]) that an equivalent description of a quasiconvex function is given by

$$
k(\beta x+(1-\beta) y) \leq \max \{k(x), k(y)\}
$$

for every $0<\beta<1$ and $x, y \in C$. By this representation it is easy to see that the class of quasiconvex functions strictly contains the class of convex functions. We now list the following result due to Sion ([14]).

Theorem 11. If the payoff function $f: A \times B \rightarrow R$ with $B$ a compact convex subset of a linear topological space and $A$ a convex subset of a linear topological space satisfies $a \longmapsto f(a, b)$ is quasiconcave and upper semicontinuous for every $b \in B$, and $b \longmapsto f(a, b)$ is quasiconvex and lower semicontinuous for every $a \in A$, then the minimax result in relation (8) given by

$$
\inf _{b \in B} \sup _{a \in A} f(a, b)=\sup _{a \in A} \inf _{b \in B} f(a, b)
$$

holds and in the above expressions inf can be replaced by max.

The following result is the starting point of the so-called level set method and shown in ([17]). Remember the values $r^{*}$ and $r_{*}$ are given in relations (1) and (2). As observed in Section 1 it is always assumed that $r^{*}>-\infty$. Also for convenience we denote the lower level set of level $r$ of a function $k: C \rightarrow R$ by

$$
L(k, r):=\{x \in C: k(x) \leq r\} .
$$

Lemma 11. Let $f: A \times B \rightarrow R$ be a given payoff function and introduce the function $f_{a}: B \rightarrow R$ given by $f_{a}(b)=f(a, b)$. Then $r^{*}=r_{*}$ if and only if $\cap_{a \in A} L\left(f_{a}, r\right)$ is nonempty for every $r>r_{*}$. 
Proof. If $r^{*}=r_{*}$, then for every $r>r_{*}=r^{*}>-\infty$ there exists by the definition of $r^{*}$ some $b_{0} \in B$ satisfying $\sup _{a \in A} f\left(a, b_{0}\right)<r$. This shows that $b_{0}$ belongs to the intersection $\cap_{a \in A} L\left(f_{a}, r\right)$ and so $\cap_{a \in A} L\left(f_{a}, r\right)$ is nonempty. To verify the reverse implication it is sufficient to check that $r^{*} \leq r_{*}+\epsilon$ for every $\epsilon>0$. Take now $r=r_{*}+\epsilon$ for some $\epsilon>0$. By our assumption we know that $\cap_{a \in A} L\left(f_{a}, r\right)$ is nonempty and so there exists some $b_{0} \in B$ satisfying $\sup _{a \in A} f\left(a, b_{0}\right) \leq r$. This implies $r^{*}=\inf _{b \in B} \sup _{a \in A} f(a, b) \leq r=r_{*}+\epsilon$ and the proof is completed.

For relation (8) to hold it is necessary and sufficient by Lemma 11 to show that the intersection $\cap_{a \in A} L\left(f_{a}, r\right)$ is nonempty for every $r>r_{*}$. It can be easily verified that for arbitrary functions $f$ this result does not hold and so we must impose some conditions on $f$. Before defining the proper class of functions we recall some well-known notions within topology. For $X$ a subset of a topological space with topology $\mathcal{F}$ the set $S \subseteq X$ is called open in $X$ if there exists some set $O$ belonging to $\mathcal{F}$ with $S=X \cap O$. The open sets generated in this way are called the relative topology induced by $X$ and with this topology the set $X$ is a topological space. Another well-known notion within topology is given in the next definition $([6],[38])$.

Definition 18. For any topological space $X$ a set $C \subseteq X$ is called connected, if for any two disjoint sets $C_{1}$ and $C_{2}$ both open (closed) in $C$ and satisfying $C=C_{1} \cup C_{2}$, it follows that $C_{1}$ or $C_{2}$ is empty.

In [40] the following class of functions is introduced.

Definition 19. Let $X$ be a topological space. The function $k: X \rightarrow R$ is called connected if for every $r \in R$ the lower level set $L(k, r) \subseteq X$ is connected.

It is well know that every convex subset of a linear topological space $X$ is connected and so any quasiconvex function $k: X \rightarrow R$ is connected. As for quasiconvex functions one can give an equivalent definition of a connected function.

Lemma 12. The function $k: X \rightarrow R$ is connected if and only if for every $x_{1}, x_{2} \in X$ there exists some connected set $C_{x_{1} x_{2}} \subseteq X$ containing $x_{1}, x_{2}$ such that $k(x) \leq \max \left\{k\left(x_{1}\right), k\left(x_{2}\right)\right\}$ for every $x \in C_{x_{1} x_{2}}$.

Proof. To show that a connected function satisfies the above property, consider $x_{1}, x_{2} \in X$ and introduce $r:=\max \left\{k\left(x_{1}\right), k\left(x_{2}\right)\right\}$. Take now the set $C_{x_{1} x_{2}}$ equal to the connected set $L(k, r)$. This set satisfies the desired property. To prove the reverse implication that the lower level sets are connected, consider some nonempty lower level set $L(k, r)$ with $x_{1}$ belonging to $L(k, r)$ and let $x_{2}$ be another arbitrary point belonging to $L(k, r)$. (The empty set is connected by definition.) By assumption there exists some connected set $C_{x_{1} x_{2}} \subseteq X$ containing $x_{1}, x_{2}$ such that

$$
k(x) \leq \max \left\{k\left(x_{1}\right), k\left(x_{2}\right)\right\}
$$


for every $x$ belonging to $C_{x_{1} x_{2}}$ This shows $C_{x_{1} x_{2}} \subseteq L(k, r)$ and since $x_{2}$ is an arbitrary element of $L(k, r)$ we obtain

$$
\cup_{x_{2} \in L(k, r)} C_{x_{1} x_{2}}=L(k, r) .
$$

By construction the intersection $\cap_{x_{2} \in L(k, r)} C_{x_{1} x_{2}}$ contains the vector $x_{1}$ and since for every $x_{2} \in L(k, r)$ the set $C_{x_{1} x_{2}}$ is connected, also $\cup_{x_{2} \in L(k, r)} C_{x_{1} x_{2}}$ is connected (cf. [38]). Applying now relation (58) shows that the function $k$ is connected.

Using the above representation of a connected function it can be show ([18]) that the set of connected functions strictly includes the set of quasiconvex functions. This means that there exist a connected function which is not quasiconvex. To prove our main theorem, we also introduce the following class of functions.

Definition 20. Let $X$ be a topological space. The collection of functions $k_{\gamma}$ : $X \rightarrow R, \gamma \in \Gamma$ is called equiconnected, if for every $x_{1}, x_{2} \in X$ there exists a connected set $C_{x_{1}, x_{2}} \subseteq X$ containing $x_{1}, x_{2}$ such that

$$
k_{\gamma}(x) \leq \max \left\{k_{\gamma}\left(x_{1}\right), k_{\gamma}\left(x_{2}\right)\right\}
$$

for every $x \in C_{x_{1} x_{2}}$ and $\gamma \in \Gamma$.

If $X$ is a convex subset of a linear topological space and for every $\gamma \in \Gamma$ the function $k_{\gamma}$ is quasiconvex, then by taking

$$
C_{x_{1} x_{2}}=\left\{\beta x_{1}+(1-\beta) x_{2}: 0 \leq \beta \leq 1\right\}
$$

it follows immediately that the collection of functions $k_{\gamma}, \gamma \in \Gamma$ is equiconnected.

Definition 21. The payoff function $f: A \times B \rightarrow R$ belongs to the class $\mathcal{C}_{0}$ if

1. The function $a \longmapsto f(a, b)$ is upper semicontinuous for every $b \in B$;

2. The function $b \longmapsto f(a, b)$ is lower semicontinuous for every $a \in A$;

3. For every $I \in \mathcal{F}(A)$ the function $b \longmapsto \max _{a \in I} f(a, b)$ is connected;

4. The collection of functions $-f_{b}, b \in B$ with $f_{b}(a):=f(a, b)$ is equiconnected.

For any set of quasiconvex functions $k_{\gamma}, \gamma \in \Gamma$ it follows that the function $x \longmapsto \sup _{\gamma \in \Gamma} k(x)$ is also quasiconvex. Using this observation it is easy to see for any payoff function $f$ satisfying $a \longmapsto f(a, b)$ is quasiconcave and upper semicontinuous for every $b \in B$ and $b \longmapsto f(a, b)$ is quasiconvex and lower semicontinuous for every $a \in A$ actually belongs to the set $\mathcal{C}_{0}$. Hence the payoff function $f$ mentioned in Sion's minimax theorem belongs to $\mathcal{C}_{0}$. One can now show the following important intersection result. 
Theorem 12. If the payoff function $f$ belongs to the class $\mathcal{C}_{0}$, then for every $r>r_{*}$ and $I \in \mathcal{F}(A)$ the intersection $\cap_{a \in I} L\left(f_{a}, r\right)$ is nonempty.

Proof. If $I=\left\{a_{0}\right\} \subseteq A$, than for every $r>r_{*}$ we obtain by the definition of $r_{*}$ that $r>\inf _{b \in B} f\left(a_{0}, b\right)$ and so $L\left(f_{a_{0}}, r\right)$ is nonempty. Suppose now for all sets $I$ belonging to $\mathcal{F}(A)$ and consisting of at most $k$ elements that

$$
\cap_{a \in I} L\left(f_{a}, r\right) \neq \emptyset
$$

for every $r>r_{*}$. To prove the result for all sets $I \in \mathcal{F}(A)$ consisting of at most $k+1$ elements we assume by contradiction that there exists some set $I_{0}=\left\{a_{0}, \ldots, a_{k}\right\} \subseteq A$ and some $r_{0}>r_{*}$ satisfying

$$
\cap_{i=0}^{k} L\left(f_{a_{i}}, r_{0}\right)=\emptyset .
$$

Since the collection of functions $-f_{b}, b \in B$ is equiconnected one can find some connected set $C_{a_{0} a_{1}} \subseteq A$ containing $a_{0}$ and $a_{1}$ satisfying

$$
f(a, b) \geq \min \left\{f\left(a_{0}, b\right), f\left(a_{1}, b\right)\right\}
$$

for every $a \in C_{a_{0} a_{1}}$ and $b \in B$. We now introduce the set valued mapping $\Phi_{r}: C_{a_{0} a_{1}} \longrightarrow 2^{B}$, given by

$$
\Phi_{r}(a)=\cap_{\gamma \in\left\{a_{2}, a_{3}, \ldots, a_{k}, a\right\}} L\left(f_{\gamma}, r\right) .
$$

(In case $k=1$ put $\Phi_{r}(a)=L\left(f_{a}, r\right)$.) By the definition of $L\left(f_{\gamma}, r\right)$ this yields

$$
\Phi_{r}(a)=\left\{b \in B: \max _{\gamma \in\left\{a_{2}, a_{3}, \ldots, a_{k}, a\right\}} f(\gamma, b) \leq r\right\} .
$$

Since the function

$$
b \longmapsto \max _{\gamma \in\left\{a_{2}, a_{3}, \ldots, a_{k}, a\right\}} f(\gamma, b)
$$

is connected and lower semicontinuous (use $b \longmapsto f(a, b)$ is lower semicontinuous for every $a \in A$ ) it follows by relation (63) that the sets $\Phi_{r}(a), a \in C_{a_{0} a_{1}}$ are connected and closed for every $r>r_{*}$. Moreover, by the induction hypothesis in relation (59) the sets $\Phi_{r_{0}}(a), a \in C_{a_{0} a_{1}}$ are nonempty and satisfy by relation $(61)$

$$
\Phi_{r_{0}}(a) \subseteq \Phi_{r_{0}}\left(a_{0}\right) \cup \Phi_{r_{0}}\left(a_{1}\right)
$$

for every $a \in C_{a_{0} a_{1}}$ and by relation (60)

$$
\Phi_{r_{0}}\left(a_{0}\right) \cap \Phi_{r_{0}}\left(a_{1}\right)=\emptyset .
$$

Introducing now the nonempty sets

$$
S_{i}:=\left\{a \in C_{a_{0} a_{1}}: \Phi_{r_{0}}(a) \subseteq \Phi_{r_{0}}\left(a_{i}\right)\right\}, i=0,1
$$

we obtain by relation (65) that the intersection $S_{0} \cap S_{1}$ is empty. To show that $S_{0} \cup S_{1}=C_{a_{0} a_{1}}$ we first observe that $S_{0} \cup S_{1} \subseteq C_{a_{0} a_{1}}$. For the reverse inclusion consider for a given $a \in C_{a_{0} a_{1}}$ the closed sets 


$$
A_{i}(a):=\Phi_{r_{0}}(a) \cap \Phi_{r_{0}}\left(a_{i}\right), i=0,1 .
$$

By relation (64) we obtain that

$$
A_{0}(a) \cup A_{1}(a)=\Phi_{r_{0}}(a)
$$

and since $\Phi_{r_{0}}(a)$ is connected it must follow by relation (67) and $A_{i}(a), i=0,1$ closed that $A_{0}(a)$ or $A_{1}(a)$ is empty. This means by relation (64) that either $\Phi_{r_{0}}(a) \subseteq \Phi_{r_{0}}\left(a_{0}\right)$ or $\Phi_{r_{0}}(a) \subseteq \Phi_{r_{0}}\left(a_{1}\right)$ and so the point $a$ belongs to $S_{0} \cup S_{1}$. Hence we have verified that the sets $S_{i}, i=0,1$ satisfy

$$
S_{0} \cap S_{1}=\emptyset, S_{0} \cup S_{1}=C_{a_{0} a_{1}} .
$$

We will now show that the sets $S_{i}, i=0,1$ are also open in $C_{a_{0} a_{1}}$. Let $a^{*}$ be an arbitrary point belonging to $S_{0}$. By our induction hypothesis we know that the sets $\Phi_{r}\left(a^{*}\right)$ are nonempty for every $r>r_{*}$ and this implies by the definition of $\Phi_{r}\left(a^{*}\right)$ in relation (63) that

$$
\inf _{b \in B} \max _{\gamma \in\left\{a_{2}, a_{3}, \ldots, a_{k}, a^{*}\right\}} f(\gamma, b) \leq r
$$

for every $r>r_{*}$. This shows by letting $r \downarrow r_{*}$ that

$$
\inf _{b \in B} \max _{\gamma \in\left\{a_{2}, a_{3}, \ldots, a_{k}, a^{*}\right\}} f(\gamma, b) \leq r_{*}<r_{0}
$$

and so one can find some $b_{0} \in \Phi_{r_{0}}\left(a^{*}\right) \subseteq B\left(b_{0} \in B\right.$ for $\left.k=1\right)$ satisfying

$$
f\left(a^{*}, b_{0}\right)<r_{0} .
$$

By the upper semicontinuity of $a \longmapsto f\left(a, b_{0}\right)$ and relation (69) there exists some open neighborhood $\mathcal{U}\left(a^{*}\right)$ of $a^{*}$ satisfying $f\left(a, b_{0}\right)<r_{0}$ for every $a \in$ $\mathcal{U}\left(a^{*}\right)$ and since $b_{0} \in \Phi_{r_{0}}\left(a^{*}\right)$ this yields $b_{0} \in \Phi_{r_{0}}(a)$ for every $a \in \mathcal{U}\left(a^{*}\right) \cap C_{a_{0} a_{1}}$ or equivalently

$$
b_{0} \in \Phi_{r_{0}}\left(a^{*}\right) \cap \Phi_{r_{0}}(a)
$$

for every $a \in \mathcal{U}\left(a^{*}\right) \cap C_{a_{0} a_{1}}$. This implies by relation (68) and $a^{*} \in S_{0}$ that $\Phi_{r_{0}}(a) \subseteq \Phi_{r_{0}}\left(a_{0}\right)$ for every $a \in \mathcal{U}\left(a^{*}\right) \cap C_{a_{0} a_{1}}$ or equivalently

$$
\mathcal{U}\left(a^{*}\right) \cap C_{a_{0} a_{1}} \subseteq S_{0} .
$$

Since $a^{*} \in S_{0}$ is arbitrary we obtain that

$$
S_{0}=\cup_{a^{*} \in S_{0}}\left(\mathcal{U}\left(a^{*}\right) \cap C_{a_{0} a_{1}}\right)=C_{a_{0} a_{1}} \cap\left(\cup_{a^{*} \in S_{0}} \mathcal{U}\left(a^{*}\right)\right)
$$

and so $S_{0}$ is open in $C_{a_{0} a_{1}}$. Similarly one can verify that the set $S_{1}$ is open in $C_{a_{0} a_{1}}$ and by relation (68) and $C_{a_{0} a_{1}}$ connected we obtain that either $S_{0}$ or $S_{1}$ is empty. Since by relation (66) the point $a_{i}$ belongs to $S_{i}, i=0,1$ this yields a contradiction, and the proof is completed.

Applying Lemma 11 we immediately deduce from Theorem 12 the following result. 
Theorem 13. Let the payoff function $f: A \times B \rightarrow R$ belong to the class $\mathcal{C}_{0}$. If $A$ is a finite set, then

$$
\inf _{b \in B} \max _{a \in A} f(a, b)=\max _{a \in A} \inf _{b \in B} f(a, b),
$$

while for $A$ an infinite set

$$
\sup _{I \in \mathcal{F}(A)} \inf _{b \in B} \max _{a \in I} f(a, b)=\sup _{a \in A} \inf _{b \in B} f(a, b) .
$$

Proof. The first formula is an immediate consequence of Lemma 11 and Theorem 12 . To verify the second formula we observe

$$
\sup _{a \in A} \inf _{b \in B} f(a, b)=\sup _{I \in \mathcal{F}(A)} \sup _{a \in I} \inf _{b \in B} f(a, b) .
$$

Applying now the first part yields the desired result.

By Theorem 13 and Lemma 3 one can show the following result, which contains as a special case (see observation after Definition 21) Sion's minimax theorem listed in Theorem 11.

Theorem 14. If $B$ is a compact topological space and the payoff function $f$ belongs to the class $\mathcal{C}_{0}$, then

$$
\inf _{b \in B} \sup _{a \in A} f(a, b)=\sup _{a \in A} \inf _{b \in B} f(a, b)
$$

and inf can be replaced by min in the above expressions.

Proof. Since $B$ is a compact topological space and $b \longmapsto f(a, b)$ is lower semicontinuous for every $a \in A$ we obtain by Lemma 3 and the observation after this lemma that

$$
\inf _{b \in B} \sup _{a \in A} f(a, b)=\sup _{I \in \mathcal{F}(A)} \inf _{b \in B} \max _{a \in I} f(a, b) .
$$

Applying now the second part of Theorem 13 and Lemma 2 yields the desired result.

Actually by Lemma 3 one can slightly weaken the condition that $A$ is a compact topological space by replacing the compactness assumption by the condition that there exists some set $I \in \mathcal{F}(A)$ such that for every $r \in R$ the set $\cap_{a \in I}\{b \in B: f(a, b) \leq r\}$ is compact. It is possible ([18]) to construct a payoff function $f$ which satisfy the conditions of Theorem 14 but does not satisfy the conditions of Sion's minimax result.

Definition 22. The payoff function $f: A \times B \rightarrow R$ belongs to the class $\mathcal{C}_{1}$ if

1. The function $a \longmapsto f(a, b)$ is upper semicontinuous for every $b \in B$;

2. The function $b \longmapsto f(a, b)$ is lower semicontinuous for every $a \in A$;

3. For every $J \in \mathcal{F}(B)$ the function $a \longmapsto \min _{b \in J} f(a, b)$ is connected;

4. The collection of functions $f_{a}, a \in A$ with $f_{a}(b):=f(a, b)$ is equiconnected. 
By the symmetry argument and Theorem 14 it follows easily that the minimax equality in relation (8) holds if the payoff function $f$ belongs to the class $\mathcal{C}_{1}$. and $A$ is a compact topological space. Finally we like to mention that Wald's minimax result is a special case of Sion's minimax result. However, from the proof of Theorem 12 it should be clear that the only properties of convex sets which are important are the observation that any intersection of convex sets is again convex and every convex set is connected. This shows that Sion's minimax result is actually a topological result based on connectedness.

\section{On n-player nonzero-sum noncooperative games}

In this section we will extend the two player zero-sum noncooperative games discussed in the previous sections to $n$-player nonzero-sum noncooperative games, $n \geq 2$. In this framework there are $n$ players and each player $i, 1 \leq$ $i \leq n$ has a pure strategy set $X_{i}$ and a payoff function $f_{i}: X \longrightarrow R$ with $X=\prod_{i=1}^{n} X_{i}$ denoting the Cartesian product of the sets $X_{i}$. In case each player $i, i=1, \ldots, n$ selects independently of each other the strategy $x_{i}$ the gain given by player $i$ is given by $f_{i}\left(x_{1}, \ldots, x_{n}\right.$ ) (For a complete description of such games and examples see [1], [3] or [10]). In this section we assume that the sets $X_{i}, 1 \leq i \leq n$ are subsets of (possibly different) linear topological spaces $\mathcal{X}_{i}([7])$. We also assume in this section that the players only use their pure strategy sets and they do not use their mixed strategy sets. For these $n$-person noncooperative games an important concept is given by a Nashequilibrium point. Observe for $n=2$ (taking $f_{2}=-f_{1}$ ) this reduces to the minimax concept used within a two player zero-sum noncooperative game.

Definition 23. Let the payoff functions $f_{i}: X \rightarrow R$ of each player be given. The point $x^{*}=\left(x_{1}^{*}, \ldots, x_{n}^{*}\right)$ is called a Nash equilibrium point if

$$
f_{i}\left(x_{1}^{*}, \ldots, x_{i}^{*}, \ldots, x_{n}^{*}\right) \geq f_{i}\left(x_{1}^{*}, \ldots, x_{i}, \ldots, x_{n}^{*}\right)
$$

for every $x_{i} \in X_{i}$ and $1 \leq i \leq n$.

We are now interested under which conditions a Nash equilibrium point exists for an $n$-person noncooperative game. To show this, we need the following definition ([41]).

Definition 24. Let $X$ be a nonempty set and $\varphi: X \times X \rightarrow R$ some function. The point $x^{*}$ is called an equilibrium point of the function $\varphi$ if $\varphi\left(x^{*}, y\right) \geq 0$ for every $y \in X$.

Using the above definition of an equilibrium point for the mapping $\varphi$, we show the following result.

Lemma 13. Let $X=\Pi_{i=1}^{n} X_{i}$ be the Cartesian product of the sets $X_{i}, i=$ $1, \ldots, n$. The point $x^{*}$ is a Nash equilibrium point if and only if $x^{*}$ is an equilibrium point of the function $\varphi: X \times X \longrightarrow R$, given by 


$$
\varphi(x, y)=\sum_{i=1}^{n} f_{i}\left(x_{1}, \ldots, x_{i}, \ldots, x_{n}\right)-f\left(x_{1}, \ldots, y_{i}, \ldots, x_{n}\right)
$$

with $x=\left(x_{1}, \ldots, x_{n}\right)$ and $y=\left(y_{1}, \ldots, y_{n}\right)$.

Proof. Let $x^{*}$ be a Nash equilibrium and consider an arbitrary $y=\left(y_{1}, \ldots, y_{n}\right) \in$ $X$. By definition

$$
f_{i}\left(x_{1}^{*}, \ldots, x_{i}^{*}, \ldots, x_{n}^{*}\right) \geq f\left(x_{1}^{*}, \ldots, y_{i}, \ldots, x_{n}^{*}\right)
$$

for every $1 \leq i \leq n$. This shows $\varphi\left(x^{*}, y\right) \geq 0$ and so $x^{*}$ is an equilibrium point of the function $\varphi$. For $x^{*}$ an equilibrium point of the function $\varphi$ consider some $1 \leq i \leq n$ and introduce the vector $y=\left(x_{1}^{*}, \ldots, y_{i}, \ldots, x_{n}^{*}\right) \in X$. Clearly for this vector $y$ it follows that

$$
0 \leq \varphi\left(x^{*}, y\right)=f_{i}\left(x_{1}^{*}, \ldots, x_{i}^{*}, \ldots, x_{n}^{*}\right)-f_{i}\left(x_{1}^{*}, \ldots, y_{i}, \ldots, x_{n}^{*}\right)
$$

and since $1 \leq i \leq n$ is arbitrary we obtain that $x^{*}$ is a Nash equilibrium point.

Hence by the above lemma we have reduced the proof of existence of a Nash equilibrium point to the proof of existence of a equilibrium point for the mapping $\varphi$ listed in relation (70). To show in general the existence of an equilibrium point of a mapping $\varphi: X \times X \longrightarrow R$ we observe that the point $x^{*}$ is an equilibrium point of the mapping $\varphi$ if and only if the intersection $\cap_{y \in X}\{x \in X: \varphi(x, y) \geq 0\}$ is nonempty. Unfortunately it seems not to be possible (in general) to prove the existence of an equilibrium point by means of LP duality or convex analysis techniques as was done for a 2-person noncooperative game. To show the existence of a Nash equilibrium under certain conditions on the sets $X_{i}$ and the payoff functions $f_{i}$ we need the socalled KKM (Knaster-Kuratowski-Mazurkiewicz) lemma ([38]). Observe the simplex $\Delta_{J}$ for any subset $J \subseteq\{1, \ldots, k\}$ is given by

$$
\Delta_{J}:=c o\left(\left\{e_{j}: j \in J\right\}\right)
$$

with $e_{j}$ the $j$ th unit vector in $R^{k}$.

Definition 25. The collection of sets $E_{i} \subseteq R^{k}, 1 \leq i \leq k$ satisfy the KKM property if $\Delta_{J} \subseteq \cup_{i \in J} E_{i}$ for every set $J \subseteq\{1, \ldots, k\}$.

The KKM lemma is given by the following result (for its proof see [37]).

Lemma 14. If the set $E_{i} \subseteq R^{k}, 1 \leq i \leq k$ are closed and satisfy the $K K M$ property then $\cap_{i=1}^{k} E_{i}$ is nonempty.

The $K K M$ lemma is an easy consequence of Sperner's lemma (see theorem 2.5.6 of [19] or Lemma 3.5.1 of [42]) and Sperner's lemma can be proved by combinatorial arguments (cf.[43] or Theorem 3.4.3 of [42]). Since our function $\varphi$ in a so-called equilibrium problem is defined on the set $X \times X$ with $X$ a convex subset of a linear topological space $\mathcal{X}$, we need to discuss the extensions 
of the KKM lemma to these spaces. This can be done in the following way. Let $\Phi: X \rightarrow 2^{X}$ be a set valued mapping with nonempty values, where $X$ is a convex subset of some (real) linear topological space $\mathcal{X}$ and $2^{X}$ the power set of $X$, and consider for a given collection $\left\{x_{1}, \ldots, x_{n}\right\} \subseteq X$ and $x \in X$ the (possibly empty) finite dimensional sets

$$
E(x)=\left\{\lambda \in \Delta_{N}: \sum_{j=1}^{n} \lambda_{j} x_{j} \in \Phi(x)\right\}
$$

with $N:=\{1, \ldots, n\}$. Denoting by $L:=\operatorname{lin}\left(\left\{x_{1}, \ldots, x_{n}\right\}\right)$ the smallest linear subspace containing the set $\left\{x_{1}, x_{2}, \ldots, x_{n}\right\}$, then clearly

$$
E(x)=\left\{\lambda \in \Delta_{N}: \sum_{j=1}^{n} \lambda_{j} x_{j} \in \Phi(x) \cap L\right\} \subseteq R^{n} .
$$

If we know that the sets $E(x) \subseteq \Delta_{N}$ are closed for every $x \in X$ and for a given collection $\left\{x_{1}, \ldots, x_{n}\right\} \subseteq X$ the nonempty sets $E_{i}:=E\left(x_{i}\right), 1 \leq i \leq n$ satisfy the KKM property, then by the KKM lemma we obtain that $\cap_{i=1}^{n} E\left(x_{i}\right)$ is nonempty. This shows that there exists some $\lambda^{*} \in \Delta_{N}$ satisfying $\sum_{j=1}^{n} \lambda_{j}^{*} x_{j} \in$ $\cap_{i=1}^{n} \Phi\left(x_{i}\right)$ and so we have verified that $\cap_{i=1}^{n} \Phi\left(x_{i}\right) \neq \emptyset$. To introduce a topology on $E(x)$ we recall the following definition.

Definition 26. The set valued mapping $\Phi: X \rightarrow 2^{X}$ with $X$ a convex subset of a linear topological space $\mathcal{X}$ is called finitely closed if for every $x \in X$ and every finite dimensional subspace $L \subseteq \mathcal{X}$ the set $\Phi(x) \cap L$ is closed in the Euclidean topology on $L$.

It is obvious that $\Phi$ finitely closed implies $E(x)$ is closed for every $x \in X$. In the next lemma we give a sufficient condition for $\Phi$ to be finitely closed.

Lemma 15. If the set-valued mapping $\Phi: X \rightarrow 2^{X}$ with $X$ a convex subset of a linear topological space $\mathcal{X}$ has closed values $\Phi(x), x \in X$, then the mapping $\Phi$ is finitely closed.

Proof. If $L \subseteq \mathcal{X}$ is a finite dimensional subspace there exists some finite set $\left\{z_{1}, \ldots, z_{n}\right\} \subseteq \mathcal{X}$ of linearly independent vectors satisfying

$$
L=\operatorname{lin}\left(\left\{z_{1}, \ldots, z_{n}\right\}\right) .
$$

To show that $\Phi(x) \cap L$ is closed in the Euclidean topology on $L$ we need to verify for any sequence $\left(x_{q}\right)_{q \in N} \subseteq \Phi(x) \cap L$ satisfying $x_{q} \rightarrow x_{\infty}$ in the Euclidean topology on $L$ that $x_{\infty} \in \Phi(x) \cap L$. Since every element of $L$ can be uniquely represented as a linear combination of the vectors $z_{i}, 1 \leq i \leq$ $n$, it follows that $x_{q} \rightarrow x_{\infty}$ in the Euclidean topology on $L$ if and only if $\lim _{q \uparrow \infty} \beta_{q}=\beta_{\infty}$ with $\beta_{q}^{\top}=\left(\beta_{q, 1}, \ldots, \beta_{q, n}\right) \in R^{n}, \beta_{\infty}^{\top}=\left(\beta_{\infty, 1}, \ldots, \beta_{\infty, n}\right) \in R^{n}$,

$$
x_{q}=\sum_{j=1}^{n} \beta_{q, j} z_{j}, q \in N,
$$

and 


$$
x_{\infty}=\sum_{j=1}^{n} \beta_{\infty, j} z_{j} .
$$

Moreover, since $\mathcal{X}$ is a linear topological space, it follows that the mapping $h: R^{n} \rightarrow X$, given by $h(\alpha)=\sum_{j=1}^{n} \alpha_{j} z_{j}$, is continuous in this topology. This shows, using $x_{q}=h\left(\beta_{q}\right) \in \Phi(x)$ for every $q \in N$, that

$$
x_{\infty}=h\left(\beta_{\infty}\right)=\lim _{q \uparrow \infty} h\left(\beta_{q}\right) \in \operatorname{cl}(\Phi(x))
$$

with the closure taken with respect to the topology on $\mathcal{X}$. Using now that $\Phi(x)$ is closed, we obtain that $x_{\infty} \in \Phi(x)$ and so $x_{\infty}$ belongs to $\Phi(x) \cap L$.

We next recall the definition of a KKM mapping for set-valued functions $\Phi: X \rightarrow 2^{X}$.

Definition 27. Let $X$ be a convex subset of a linear topological space $\mathcal{X}$. The set valued mapping $\Phi: X \rightarrow 2^{X}$ is called a KKM mapping if $c o\left(\left\{x_{1}, \ldots, x_{k}\right\}\right) \subseteq$ $\cup_{j=1}^{k} \Phi\left(x_{j}\right)$ for every finite subset $\left\{x_{1}, \ldots, x_{k}\right\} \subseteq X$.

Clearly by the above definition it follows for a KKM mapping $\Phi$ that $x$ belongs to $\Phi(x)$ for every $x \in X$. In the next lemma we extend the KKM lemma to set-valued mappings.

Lemma 16. If the set valued mapping $\Phi: X \rightarrow 2^{X}$ is a KKM mapping with $\Phi(x)$ closed for every $x \in X$, then $\cap_{i=1}^{k} \Phi\left(x_{i}\right)$ is nonempty for every finite set $\left\{x_{1}, \ldots, x_{k}\right\} \subseteq X$.

Proof. If $\Phi$ is a KKM mapping then by definition

$$
c o\left(\left\{x_{1}, \ldots, x_{k}\right\}\right) \subseteq \cup_{j=1}^{k} \Phi\left(x_{j}\right)
$$

for every finite subset $\left\{x_{1}, \ldots, x_{k}\right\} \subseteq X$. To prove the desired result we verify by induction that

$$
c o\left(\left\{x_{1}, \ldots, x_{q}\right\}\right) \cap\left(\cap_{j=1}^{q} \Phi\left(x_{j}\right)\right) \neq \emptyset .
$$

for every finite subset $\left\{x_{1}, \ldots, x_{q}\right\} \subseteq X$. By relation (74) it follows that (75) holds for $q=1$. Suppose now that relation (75) holds for $q \leq k-1(k \geq 2)$ and consider a subset $\left\{x_{1}, \ldots, x_{k}\right\} \subseteq X$. Let $\Delta_{k}:=\left\{\lambda \in R^{k}: \lambda_{i} \geq 0, \sum_{i=1}^{k} \lambda_{i}=1\right\}$ and introduce for every $1 \leq i \leq k$ the sets $E_{i}$, given by

$$
E_{i}=\left\{\lambda \in \Delta_{k}: \sum_{j=1}^{k} \lambda_{j} x_{j} \in \Phi\left(x_{i}\right)\right\} \subseteq R^{k} .
$$

For $L$ denoting the linear subspace $\operatorname{lin}\left(\left\{x_{1}, \ldots, x_{k}\right\}\right)$ it is obvious that

$$
E_{i}=\left\{\lambda \in \Delta_{k}: \sum_{j=1}^{k} \lambda_{j} x_{j} \in \Phi\left(x_{i}\right) \cap L\right\},
$$

and since by Lemma 15 the set valued mapping $\Phi$ is finitely closed it follows that the sets $E_{i}, 1 \leq i \leq k$ are closed in the Euclidean topology on $L$. 
Moreover, to show that the sets $E_{i}, 1 \leq i \leq k$, satisfy the KKM property we observe for every $J \subseteq\{1, . ., k\}$ and $\lambda \in \operatorname{co}\left(\left\{e_{j}: j \in J\right\}\right) \subseteq \Delta_{k}$ that

$$
\lambda=\left(\lambda_{1}, \ldots, \lambda_{k}\right), \lambda_{j}=0, j \notin J, \lambda_{j} \geq 0, j \in J, \sum_{j \in J} \lambda_{j}=1 .
$$

This implies by relation (74) with $k$ replaced by $|J|$ that

$$
\sum_{j=1}^{k} \lambda_{j} x_{j}=\sum_{j \in J} \lambda_{j} x_{j} \in \operatorname{co}\left(\left\{x_{j}: j \in J\right\}\right) \subseteq \cup_{j \in J} \Phi\left(x_{j}\right)
$$

and we have verified that $\lambda$ belongs to $\cup_{j \in J} E_{j}$. Since $\lambda \in c o\left(\left\{e_{j}: j \in J\right\}\right)$ is arbitrary this shows that

$$
c o\left(\left\{e_{j}: j \in J\right\}\right) \subseteq \cup_{j \in J} E_{j}
$$

and so the collection $E_{i}, 1 \leq i \leq k$ satisfies the KKM property. Hence by the $K K M$ lemma it follows that $\cap_{i=1}^{k} E_{i}$ is nonempty and so there exists some $\lambda^{*} \in \Delta_{k}$ satisfying $\sum_{j=1}^{k} \lambda_{j}^{*} x_{j} \in \cap_{i=1}^{k} \Phi\left(x_{i}\right)$. This proves the induction for $k$ and the proof is completed.

We are now able to show that under certain conditions a Nash equilibrium point exists. To prove this, we first need the following lemma.

Lemma 17. Let $X$ be a convex subset of a linear topological space $\mathcal{X}$. If the function $\varphi: X \times X \rightarrow R$ satisfies $\varphi(x, x) \geq 0$ and $y \longmapsto \varphi(x, y)$ is convex on $X$ for every $x \in X$, then the set valued mapping $\Phi: X \longrightarrow 2^{X}$ given by

$$
\Phi(y)=\{x \in X: \varphi(x, y) \geq 0\}
$$

is a KKM mapping.

Proof. Since $\varphi(x, x) \geq 0$ it follows immediately that $y$ belongs to $\Phi(y)$. Suppose now by contradiction that there exists some finite set $\left\{y_{1}^{*}, \ldots, y_{k}^{*}\right\} \subseteq$ $X, k \geq 2$ such that $y^{*}$ belonging to $\operatorname{co}\left(\left\{y_{1}^{*}, \ldots, y_{k}^{*}\right\}\right)$ does not belong to $\cup_{j=1}^{k} \Phi\left(y_{j}^{*}\right)$. By the first part it follows that $y^{*}$ is not equal to $y_{i}^{*}$ for some $1 \leq i \leq k$. This means that one can find some $\lambda^{*} \in \Delta_{k}$ with at least two positive components smaller than 1 satisfying

$$
\max _{1 \leq i \leq k} \varphi\left(\sum_{j=1}^{k} \lambda_{j}^{*} y_{j}^{*}, y_{i}^{*}\right)<0 .
$$

By the convexity of the function $y \longmapsto \varphi\left(\sum_{j=1}^{k} \lambda_{j}^{*} y_{j}^{*}, y\right)$ this implies

$$
0 \leq \varphi\left(\sum_{j=1}^{k} \lambda_{j}^{*} y_{j}^{*}, \sum_{i=1}^{k} \lambda_{i}^{*} y_{i}^{*}\right) \leq \sum_{i=1}^{k} \lambda_{i}^{*} \varphi\left(\sum_{j=1}^{k} \lambda_{j}^{*} y_{j}, y_{i}^{*}\right)<0
$$

and we obtain a contradiction.

Finally we can give a proof of the following important result. 
Theorem 15. If the pure strategy sets $X_{i}, 1 \leq i \leq n$ are convex compact subsets of (maybe different) linear topological spaces $\mathcal{X}_{i}$, the payoff functions $f_{i}: X \rightarrow R, 1 \leq i \leq n$ are continuous on $X$ for every $1 \leq i \leq n$ and satisfy

$$
x_{i} \longmapsto f_{i}\left(x_{1}, \ldots, x_{i}, \ldots, x_{n}\right)
$$

are concave for every $1 \leq i \leq n$ and every fixed $\left(x_{1}, \ldots, x_{i-1}, x_{i+1}, \ldots, x_{n}\right)$, then the n-person noncooperative game has a Nash equilibrium point.

Proof. By Lemma 13 we have to show for $X=\prod_{i=1}^{n} X_{i}$ that the function $\varphi: X \times X \longrightarrow R$, given by

$$
\varphi(x, y)=\sum_{i=1}^{n} f_{i}\left(x_{1}, . ., x_{i}, . ., x_{n}\right)-f_{i}\left(x_{1}, \ldots, y_{i}, \ldots, x_{n}\right)
$$

with $x=\left(x_{1}, \ldots, x_{n}\right)$ and $y=\left(y_{1}, \ldots, y_{n}\right)$ has an equilibrium point, and by the observations after Lemma 13 this means that $\cap_{y \in X} \Phi(y)$ is nonempty with $\Phi(y):=\{x \in X: \varphi(x, y) \geq 0\}$. First observe by the continuity of $f_{i}(1 \leq$ $i \leq n)$ that the function $x \longmapsto \varphi(x, y)$ listed in relation (76) is continuous on $X$ for every $y \in X$. This shows for every $y \in X$ that the set $\Phi(y)$ is closed and since $X$ is compact that $\Phi(y)$ is compact as well. Moreover, since $x_{i} \longmapsto f_{i}\left(x_{1}, \ldots, x_{i}, \ldots, x_{n}\right)$ is concave for every $1 \leq i \leq n$, we obtain that the function $y \longmapsto \varphi(x, y)$ is convex and together with $\varphi(x, x)=0$ this implies by Lemma 17 that the set-valued map $\Phi$ is a KKM map. Applying now Lemma 16 it follows for every finite subset $F \subseteq X$ that $\cap_{y \in F} \Phi(y)$ is nonempty. This shows by the finite intersection property for compact sets that $\cap_{y \in X} \Phi(y)$ is nonempty, and we have shown the desired result.

\section{References}

1. Başar T, Olsder GJ (1999) Dynamic noncooperative game theory (second edition), SIAM, Philadelphia

2. Kuhn HW (2003) Lectures on the theory of games, Princeton University Press, Princeton

3. Vorob'ev N.N (1977) Game theory: lectures for economists and systems scientists, Springer, New York

4. Jones AJ (2000) Game theory: mathematical models of conflict, Horwood Publishing, Chicester

5. Aubin JP (1979) Mathematical methods of game and economic theory, North Holland, Amsterdam

6. Royden HL (1988) Real analysis (third edition), Macnmillan Publishing Company, New York

7. Rudin W (1991) Functional analysis, McGraw-Hill, New York

8. Choquet G (1976) Lectures on analysis volume 1: Integration and topological vector spaces, W.A.Benjamin, Reading

9. Dudley RM (1989) Real Analysis and Probability, Wadsworth and Brooks/Cole, Pacific Grove 
10. Szëp J, Forgó F (1985) Introduction to the theory of games, Akadémia Kiadó, Budapest

11. Rockafellar RT (1972) Convex analysis, Princeton University Press, Princeton

12. Nash J (1950) Equilibrium points in n-person games, Proc.Natl.Acad.Sci. USA $36: 48-49$

13. Nash J (1951) Non-cooperative games, Ann. of Math. 54:286-295

14. Sion M (1958) On general minmax theorems, Pacific J.Math 8:171-176

15. von Neumann J (1928) Zur theorie der gesellschaftsspiele,Math.Ann. 100:295320

16. Kjeldsen TH (2001) John van Neumann's conception of the minimax theorem: a journey through different mathematical concepts, Arch.Hist.Exact Sci. 56:3968

17. Joó I (1989) On some convexities, Acta Math. Hung. 54(1-2):163-172

18. Frenk JBG, Kassay G (2006) The level set method of Joó and its use in minmax theory, Mathematical Programming Ser.A 105:145-155

19. Yang Z (1996) Simplicial Fixed Point Algorithms and Applications. Phd Thesis, Center for Economic Research, University of Tilburg, Tinburg

20. Rudin W (1976) Principles of Mathematical Analysis, McGraw-Hill, New York

21. Wald A (1945) Generalization of a theorem by von Neumann concerning zerosum two-person games, Annals of Mathematics 46 (2):281-286

22. Chv'atal V (1999) Linear Programming, Freeman, New York

23. von Neumann J, Morgenstern O, (1944) Theory of games and economic behavior, Princeton University Press, Princeton

24. Kneser H (1952) Sur un théorème fondamental de la théorie des jeux, Comptes Rendus Acad.Sci.Paris 234: 2418-2420

25. Frenk JBG, Kassay G, Kolumbán J (2004) On equivalent results in minimax theory, European Journal of Operational Research 157:46-58

26. Ville J (1938) Sur la théorie générale des jeux au intervient l'habilité des jouers. Traité du calcul des probabilités et de ses applications, Edited by E.Borel et al., Gauthier-Villars Cie, Paris, 105-113.

27. Kassay G, Kolumbán, J (1996) On a generalized sup-inf problem, Journal of Optimization Theory and Applications 91:651-670

28. Kolmogorov AN, Fomin SV (1975) Introductory real Analysis, Dover Publications, New York

29. Frenk JBG, Kassay G, Protasov V (2005) On Borel probability measures and noncooperative game theory, Optimization 54:81-101

30. Frenk JBG, Kas P, Kassay G (2006) On linear programming duality and necessary and sufficient conditions in minimax theory, to appear in Journal of Optimization Theory and Applications

31. Simons S (1995), Minimax theorems and their proofs. In Minimax theorems and their applications, Edited by Du DZ and Pardalos PM, Kluwer Academic Publishers, Dordrecht, 1-23

32. Fan K (1953) Minimax theorems, Proc.Nat.Acad.Sci.U.S.A. 39:42-47

33. König H (1968) Uber das von Neumannsche minimax-theorem, Arch.Math 19:482-487

34. Frenk JBG, Kassay G (2002) Minimax results and finite dimensional separation, Journal of Optimization Theory and Applications 113 (2):409-421

35. Jeyakumar V (1986) A generalization of a minimax theorem of Fan via a theorem of the alternative, Journal of Optimization Theory and Applications 48 (3):525-533 
36. Green JW, Austin W (1950) Quasiconvex sets, Canadian J.Math. 2:489-507

37. Yuan GXZ (1999) KKM theory and applications in nonlinear analysis, Marcel Dekker, New York

38. Engelking R (1968) Outline of general topology, North-Holland, Amsterdam

39. Avriel M, Diewert WE, Schaible S, Zang I (1988) Generalized concavity, Plenum Press, New York

40. Martin DH (1982) Connected level sets, minimizing sets and uniqueness in optimization, Journal of Optimization Theory and Applications 36(1):71-91

41. Blum E, Oettli W (1994) From optimization and variational inequalities to equilibrium problems, The Mathematical Student 63 no:1-4:123-145

42. van Mill J (1989) Infinite Dimensional Topology: Prerequisites and Introduction, North-Holland, Amsterdam

43. Aigner M, Ziegler GM (1999) Proofs from THE BOOK, Springer, New York 